\title{
Hydromechanics of low-Reynolds-number flow. Part 2. Singularity method for Stokes flows
}

\author{
By ALLEN T. CHWANG AND T. YAO-TSU WU
}

Engineering Science Department, California Institute of Technology, Pasadena

(Received 18 March 1974)

The present study further explores the fundamental singular solutions for Stokes flow that can be useful for constructing solutions over a wide range of free-stream profiles and body shapes. The primary singularity is the Stokeslet, which is associated with a singular point force embedded in a Stokes flow. From its derivatives other fundamental singularities can be obtained, including rotlets, stresslets, potential doublets and higher-order poles derived from them. For treating interior Stokes-flow problems new fundamental solutions are introduced; they include the Stokeson and its derivatives, called the roton and stresson.

These fundamental singularities are employed here to construct exact solutions to a number of exterior and interior Stokes-flow problems for several specific body shapes translating and rotating in a viscous fluid which may itself be providing a primary flow. The different primary flows considered here include the uniform stream, shear flows, parabolic profiles and extensional flows (hyperbolic profiles), while the body shapes cover prolate spheroids, spheres and circular cylinders. The salient features of these exact solutions (all obtained in closed form) regarding the types of singularities required for the construction of a solution in each specific case, their distribution densities and the range of validity of the solution, which may depend on the characteristic Reynolds numbers and governing geometrical parameters, are discussed.

\section{Introduction}

The hydromechanies of low-Reynolds-number flows play an important role in the study of rheology, lubrication theory, micro-organism locomotion and many areas of biophysical and geophysical interest. In the case when the inertial effects are negligible compared with the viscous forces, the Navier-Stokes equations are usually simplified to the Stokes equations as a first approximation. Determination of the solutions for the Stokes flows, however, is still recognized to be difficult in general for arbitrary body shapes. As a consequence, not many exact solutions are known.

Of the few analytical methods available for solving Stokes-flow problems, one is the boundary-value method, which is based on the choice of an appropriate c0-ordinate system to facilitate separation of the variables for the body geometry in question. Another is the singularity method, whose accuracy depends largely on whether the correct types of singularity are used and how their spatial distributions are chosen. The boundary-value method seems to have been widely adopted 
in practice, more so than the singularity method. In the literature, the most important exact solutions of Stokes-flow problems are those found by using the classical treatment of the motion of ellipsoids by Oberbeck (1876), Edwardes (1892) and Jeffery (1922) (see also Lamb 1932, p. 604); all these studies are based on the use of ellipsoidal co-ordinates and on some rather sophisticated analysis of ellipsoidal harmonics. In contrast, these solutions have not been derived before by means of the singularity method.

Actually the singularity method has been known since the pioneering work of Lorentz (1897), Oseen (1927) and Burgers (1938). It has been further developed and applied in the recent studies of slender-body theory for low-Reynoldsnumber flows by Hancock (1953), Broersma $(1960)$, Tuck $(1964,1970)$, Taylor (1969), Batchelor $(1970 a, b)$, Tillett (1970), Cox (1970, 1971), Blake \& Chwang (1974) and others. Through these investigations the relative simplicity and effectiveness of the method have gradually become more recognized. Nevertheless, it is felt that the potential power of the singularity method has not been fully explored for the general case of arbitrary body shapes as well as for the special case of slender bodies. The primary difficulty is the lack of general knowledge about the types of singularity required and their distribution densities, which are dictated by the specific body shape and different free-stream velocity profiles. It is thought that further development of the method can be greatly enhanced by accumulating a number of exact solutions for several representative cases, since useful information could be extracted from these solutions to guide more general theories.

Following this objective, the first part (Chwang \& Wu 1974a) of this series has been devoted to a study of the purely rotational flow generated by the rotation of axisymmetric prolate bodies of various shapes about their longitudinal axes. In the present part we shall investigate the translational and rotational motion of prolate spheroids, spheres and circular cylinders in Stokes flow for several different free-stream velocity profiles. In $\$ 2$ we begin with a discussion of the fundamental singular solutions of Stokes flow, including a Stokeslet and its derivatives known as rotlets, stresslets, potential doublets and higher-order poles derived from them. Also introduced here are new fundamental solutions called Stokesons, rotons and stressons; they are useful for constructing solutions of interior flow problems as well as for representing a local free stream having a shear or an 'extensional flow' field. These fundamental solutions are then employed to construct the exact solutions to a number of problems described by the titles of $\S \S 3-14$. From these examples we note that the singularity method may have further advantages once the basic properties of different singularities are clarified. The method can be effectively executed with a set of Cartesian co-ordinates in general. Derivation of the net force and torque on the body is especially simple, as they can be determined by a direct integration of the distributed Stokeslets and rotlets, not requiring an integration of the surface stresses as in the boundary-value method. The important features of these exact solutions are discussed, especially with respect to the types of singularity required for the construction of a solution in different situations, and to the distribution density of these singularities. 
Further attempts are made here to use these exact solutions, all being expressed in closed form, to provide an estimate of their range of validity, which may be affected by the characteristic Reynolds numbers and the body geometry. The latter effect, as has been noted, has not been thoroughly explored for lowReynolds-number flows in general. Possible improvements to the solution beyond the range of validity of the Stokes approximation will be discussed in a future paper.

\section{Fundamental solutions of the Stokes equations}

The starting point of the singularity method is the inhomogeneous Stokes equations

$$
\nabla \cdot \mathbf{u}=0, \quad \nabla p=\mu \nabla^{2} \mathbf{u}+\mathbf{f},
$$

where $\mathbf{u}$ is the velocity vector, $p$ the pressure, $\mu$ the constant viscosity coefficient, and $\mathbf{f}(\mathbf{x})$ the external force per unit volume, $\mathbf{x}$ being the position vector in a threedimensional Euclidean space. The class of incompressible viscous flows considered here is assumed to have negligible inertial effects when the Reynolds number is sufficiently low. (Consistent with this assumption, the external force $f$ may also depend on the time $t$, as a parameter, provided that it varies sufficiently slowly with $t$.) The solutions of (1) corresponding to forces having a certain singular behaviour in an unbounded flow will be called the fundamental solutions, and such forces, the fundamental singularities. We discuss below the important cases with the purpose of compiling a set of necessary 'building blocks' for the eventual construction of solutions of various general boundary-value problems.

The primary fundamental solution is associated with a singular point force located, say, at the origin,

$$
\mathbf{f}_{S}=8 \pi \mu \alpha \delta(\mathbf{x}),
$$

$\boldsymbol{\alpha}$ being a constant vector and $\delta(\mathbf{x})$ the three-dimensional Dirac delta-function. It is called a Stokeslet, after Hancock (1953), and $\alpha$ characterizes its strength (in magnitude and direction). The solution of (1) with $\mathbf{f}=\mathbf{f}_{S}$ can be derived in a quite straightforward manner. (As a direct approach, $p$ can be readily found from the equation obtained by taking the divergence of $(1 b)$, the vorticity vector from the curl of $(1 b)$, and the velocity can be determined upon integration of either the vorticity or $(1 b)$ under condition $(1 a)$.) Thus the velocity $\mathrm{U}_{S}$, pressure $P_{S}$ and vorticity $\zeta_{S}=\nabla \times U_{S}$ of a Stokeslet of strength $\alpha$ are

$$
\begin{aligned}
& \mathrm{U}_{S}(\mathbf{x} ; \alpha)=\alpha / R+(\alpha \cdot \mathbf{x}) \mathbf{x} / R^{3} \quad(R=|\mathbf{x}|), \\
& P_{S}(\mathbf{x} ; \alpha)=-2 \mu \nabla \cdot(\alpha / R)=2 \mu \alpha \cdot \mathbf{x} / R^{3} \\
& \zeta_{S}(\mathbf{x} ; \alpha)=2 \nabla \times(\alpha / R)=2 \alpha \times \mathbf{x} / R^{3}
\end{aligned}
$$

Here the pressure is measured, for simplicity, relative to the pressure $p_{\infty}$ at infinity. It is noted that the velocity field of a Stokeslet has a long-range effect, falling off like $R^{-1}$ at large distances, while the pressure $P_{S}$ and vorticity $\zeta_{S}$ both 
decrease like $R^{-2}$. The total force exerted by a Stokeslet on the fluid outside a control surface $S_{c}$ enclosing the Stokeslet is given by

$$
\mathbf{F}=\int_{S_{\mathrm{e}}}(p \mathbf{n}-\boldsymbol{\tau} \mathbf{n}) d S=\int_{V_{\mathrm{e}}}\left(\nabla P_{S}-\mu \nabla^{2} \mathbf{U}_{S}\right) d V=\int_{V_{\mathrm{e}}} \mathbf{f}_{S} d V=8 \pi \mu \boldsymbol{\alpha},
$$

where $\mathbf{n}$ is the unit outward normal at $S_{c}$ and $\tau$ is the viscous stress tensor. The second step indicates an application of the divergence theorem to the volume $V_{c}$ within $S_{c}$, and the last two steps follow from $(1 b)$ and (2).

Obviously, a derivative of any order of $\mathbf{U}_{S}$ and $P_{S}$ is also a solution of (1), the corresponding $\mathbf{f}$ being the derivative of the same order of $\mathbf{f}_{S}$. These derivatives are readily obtained from the 'formal' multipole expansion of a Stokeslet at $\mathbf{x}=\boldsymbol{\xi}$ in a Taylor series about $\mathbf{x}$ :

$$
\mathbf{U}_{S}(\mathbf{x}-\xi)=\mathbf{U}_{S}(\mathbf{x})-(\xi . \nabla) \mathbf{U}_{S}(\mathbf{x})+\frac{1}{2}(\xi . \nabla)^{2} \mathbf{U}_{S}(\mathbf{x})+\ldots,
$$

together with similar expansions for $P_{S}$ and $\zeta_{S}$. With an appropriate interpretation of the multipole moments, as is generally practised in potential theory, we may introduce the Stokes doublet, Stokes quadrupole, etc., as

$$
\begin{aligned}
& \mathrm{U}_{S D}(\mathbf{x} ; \boldsymbol{\alpha}, \boldsymbol{\beta})=-(\boldsymbol{\beta} . \nabla) \mathrm{U}_{S}(\mathbf{x} ; \boldsymbol{\alpha}) \\
& =\frac{(\boldsymbol{\beta} \times \boldsymbol{\alpha}) \times \mathbf{x}}{R^{3}}-\left[\frac{(\alpha \cdot \beta) \mathbf{x}}{R^{3}}-3 \frac{(\alpha \cdot \mathbf{x})(\boldsymbol{\beta} \cdot \mathbf{x}) \mathbf{x}}{-R^{5}}\right], \\
& P_{S D}(\mathbf{x} ; \boldsymbol{\alpha}, \boldsymbol{\beta})=-(\boldsymbol{\beta} . \nabla) P_{S}(\mathbf{x} ; \boldsymbol{\alpha})=2 \mu\left[-\frac{\alpha \cdot \boldsymbol{\beta}}{R^{3}}+3 \frac{(\boldsymbol{\alpha} \cdot \mathbf{x})(\boldsymbol{\beta} \cdot \mathbf{x})}{R^{5}}\right] \text {, } \\
& \mathbf{U}_{S 4}(\mathbf{x} ; \boldsymbol{\alpha}, \boldsymbol{\beta}, \boldsymbol{\gamma})=(\boldsymbol{\gamma}, \nabla)(\boldsymbol{\beta} . \nabla) \mathrm{U}_{\mathrm{S}}(\mathbf{x} ; \boldsymbol{\alpha}), \\
& P_{S 4}(\mathbf{x} ; \alpha, \beta, \gamma)=(\gamma . \nabla)(\beta . \nabla) P_{S}(\mathbf{x} ; \alpha),
\end{aligned}
$$

where $\alpha, \beta$ and $\gamma$ are constant vectors constituting the pole moments. The velocities of these Stokes multipoles, while vector functions of $\mathbf{x}$, may be regarded as tensorial quantities in terms of the (Cartesian) components $\alpha_{i}, \beta_{j}$ and $\gamma_{k}$ of $\alpha, \beta$ and $\gamma$. Although each of these tensorial components of $(U, P)$ is itself a solution of the Stokes equations, it is often useful, for the interpretation of their physical significance, to group them in certain combinations.

For instance, the antisymmetric component (with respect to an interchange of $\alpha$ and $\beta$ ) of a Stokes doublet [see (6a)] is itself a physical entity; it is a rotlet (also called a couplet by Batchelor 1970 $a$ ) and its velocity, pressure and vorticity are given by

$$
\begin{aligned}
\mathrm{U}_{R}(\mathbf{x} ; \boldsymbol{\gamma}) & =\frac{1}{2}\left[\mathrm{U}_{S D}(\mathbf{x} ; \boldsymbol{\beta}, \boldsymbol{\alpha})-\mathrm{U}_{S D}(\mathbf{x} ; \boldsymbol{\alpha}, \boldsymbol{\beta})\right]=\frac{1}{2} \nabla \times \mathrm{U}_{S}(\mathbf{x} ; \boldsymbol{\gamma}) \\
& =\boldsymbol{\gamma} \times \mathbf{x} / R^{3} \quad(\boldsymbol{\gamma}=\boldsymbol{\alpha} \times \boldsymbol{\beta}), \\
P_{R}(\mathbf{x} ; \boldsymbol{\gamma}) & =\frac{1}{2}\left[P_{S D}(\mathbf{x} ; \boldsymbol{\beta}, \boldsymbol{\alpha})-P_{S D}(\mathbf{x} ; \boldsymbol{\alpha}, \boldsymbol{\beta})\right]=0, \\
\zeta_{R}(\mathbf{x} ; \boldsymbol{\gamma}) & =\nabla \times \mathrm{U}_{R}=\nabla \times(\nabla \times(\gamma / R))=\nabla(\nabla \cdot(\boldsymbol{\gamma} / R))+4 \pi \gamma \delta(\mathbf{x}) .
\end{aligned}
$$

The singular forcing function of a rotlet is clearly

$$
\mathbf{f}_{R}=4 \pi \mu[(\alpha . \nabla) \beta-(\beta . \nabla) \alpha] \delta(\mathbf{x})=4 \pi \mu \nabla \times(\gamma \delta(\mathbf{x})) .
$$


The above solution (7) can also be derived directly from (1) with $\mathbf{f}=\mathbf{f}_{R}$ (see part 1). Physically, a rotlet flow may be regarded as that due to a singular point torque at the origin, since the moment exerted on the fluid by a rotlet of strength $\gamma$ is

$$
\mathbf{M}=\int_{S_{c}} \mathbf{x} \times(p \mathbf{n}-\boldsymbol{\tau n}) d S=\int_{V_{c}} \mathbf{x} \times \mathbf{f}_{R} d V=8 \pi \mu \boldsymbol{\gamma},
$$

for any closed control surface $S_{c}$ containing the rotlet. On the other hand, the net force on the fluid due to a rotlet is zero.

The symmetric component of a Stokes doublet gives a fundamental singularity called a stresslet, after Batchelor $(1970 a)$. Its velocity, pressure and vorticity [see $(6 a, b)]$ are

$$
\begin{aligned}
& \mathbf{U}_{S S}(\mathbf{x} ; \alpha, \beta)=\left[-\frac{\alpha \cdot \beta}{R^{3}}+\frac{3(\alpha \cdot \mathbf{x})(\beta \cdot \mathbf{x})}{R^{5}}\right] \mathbf{x} \\
& P_{S S}(\mathbf{x} ; \alpha, \beta)=2 \mu\left[-\frac{\alpha \cdot \beta}{R^{3}}+\frac{3(\alpha \cdot \mathbf{x})(\beta \cdot \mathbf{x})}{R^{5}}\right], \\
& \zeta_{S S}(\mathbf{x} ; \alpha, \beta)=\left(3 / R^{5}\right)[(\beta . \mathbf{x}) \alpha+(\alpha \cdot \mathbf{x}) \beta] \times \mathbf{x} .
\end{aligned}
$$

The stresslet velocity $\mathbf{U}_{S S}$ can also be expressed in a tensorial form with its strength characterized by a symmetric second-rank tensor (Batchelor 1970a). Physically, a stresslet represents straining motion of the fluid symmetric about the $\alpha, \beta$ plane with the principal axes of strain lying in the $\alpha+\beta, \alpha-\beta$ and $\alpha \times \beta$ directions. In virtue of this symmetry, a stresslet contributes no net force or moment to the fluid.

A potential doublet is well known to possess the velocity field

$$
\mathbf{U}_{D}(\mathbf{x} ; \boldsymbol{\delta})=\nabla\left(\nabla \cdot \frac{\delta}{R}\right)=-\frac{\delta}{R^{3}}+\frac{3(\boldsymbol{\delta} \cdot \mathbf{x}) \mathbf{x}}{R^{5}}
$$

$\delta$ being the doublet strength. It is of interest, however, to note that a potential doublet is related to a Stokeslet by

$$
\mathbf{U}_{D}(\mathbf{x} ; \boldsymbol{\delta})=-\frac{1}{2} \nabla^{2} \mathbf{U}_{S}(\mathbf{x} ; \boldsymbol{\delta}) \quad(0<|\mathbf{x}|<\infty) .
$$

In accordance with this relationship, the corresponding pressure, now under the assumption of negligible inertial effects, is therefore

$$
P_{D}(\mathbf{x} ; \boldsymbol{\delta})=-\frac{1}{2} \nabla^{2} P_{S}(\mathbf{x} ; \boldsymbol{\delta})=0 \quad(0<|\mathbf{x}|<\infty),
$$

since $P_{S}(\mathbf{x})$ is a harmonic function of $\mathbf{x}$. Thus we see that, while the potential doublet retains its kinematic identity with a doublet in potential flow, its dynamic effect now diminishes as the inertial effects become negligible. Further, since the potential doublet is a derivative of a Stokeslet, hence so are all the higher-order poles of potential flow derived from them. Regarding its utility for constructing solutions, the potential doublet plays a fundamental role in potential theory as well as in low-Reynolds-number flow problems. Various potential problems can be solved by employing an appropriate spatial distribution of doublets only (see, for example, Chwang \& Wu 1974b). These potential-flow 
solutions have provided valuable suggestions about the basic structure of the singularity solutions of analogous Stokes-flow problems to be presented later.

For interior Stokes-flow problems it is useful to seek solutions of (1) that are singular at infinity but regular everywhere else. Such a fundamental solution which has a quadrupole at infinity will be called a 'Stokeson' and, more specifically, is given by

$$
\begin{aligned}
& \mathbf{U}_{S N}(\mathbf{x} ; \alpha)=2 R^{2} \alpha-(\alpha . \mathbf{x}) \mathbf{x}, \\
& P_{S N}(\mathbf{x} ; \alpha)=10 \mu(\alpha \cdot \mathbf{x}), \\
& \zeta_{S N}(\mathbf{x} ; \alpha)=\nabla \times \mathbf{U}_{S N}=-5 \alpha \times \mathbf{x} .
\end{aligned}
$$

A 'Stokeson dipole' is a derivative of a Stokeson; it has a velocity linear in $\mathbf{x}$ and a uniform vorticity and is given by

$$
\begin{aligned}
& \mathrm{U}_{N D}(\mathbf{x} ; \boldsymbol{\alpha}, \boldsymbol{\beta})=-(\boldsymbol{\beta} . \nabla) \mathrm{U}_{S N}(\mathbf{x} ; \boldsymbol{\alpha})=(\boldsymbol{\alpha} \cdot \boldsymbol{\beta}) \mathbf{x}+(\boldsymbol{\alpha} \cdot \mathbf{x}) \boldsymbol{\beta}-4(\boldsymbol{\beta} \cdot \mathbf{x}) \boldsymbol{\alpha}, \\
& P_{N D}(\mathbf{x} ; \boldsymbol{\alpha}, \boldsymbol{\beta})=-10 \mu(\boldsymbol{\alpha} \cdot \boldsymbol{\beta}) \\
& \zeta_{N D}(\mathbf{x} ; \boldsymbol{\alpha}, \boldsymbol{\beta})=5 \boldsymbol{\alpha} \times \boldsymbol{\beta} .
\end{aligned}
$$

A particular solution of this class useful for representing rotational flows at low Reynolds numbers is the antisymmetric component of $\mathbf{U}_{N D}$, which we shall call a 'roton':

$$
\mathrm{U}_{R N}=\gamma \times \mathbf{x}, \quad P_{R N}=0, \quad \zeta_{R N}=2 \gamma .
$$

Thus a roton represents a rigid-body rotation, in which the pressure due to the centrifugal inertia effect is neglected in accordance with the basic assumption. Another particular solution which is useful for describing local straining motion is the symmetric part of a Stokeson dipole, which we shall call a 'stresson'. It has the velocity

$$
\mathbf{U}_{S S N}=(\boldsymbol{\alpha} \cdot \boldsymbol{\beta}) \mathbf{x}-\frac{3}{2}[(\boldsymbol{\alpha} \cdot \mathbf{x}) \boldsymbol{\beta}+(\boldsymbol{\beta} \cdot \mathbf{x}) \boldsymbol{\alpha}],
$$

which is irrotational, and carries no pressure variation. A stresson is seen to consist of a shear-strain component (in Cartesian form)

$$
u=A_{12} y+A_{13} z, \quad v=A_{12} x+A_{23} z, \quad w=A_{13} x+A_{23} y,
$$

and a principal straining motion

$$
u=a x, \quad v=b y, \quad w=c z \quad(a+b+c=0) .
$$

The latter is known as an 'extensional flow' in rheology. It becomes an exact solution of the Navier-Stokes equations if it is associated with the pressure $p=-\frac{1}{2} \rho\left(a^{2} x^{2}+b^{2} y^{2}+c^{2} z^{2}\right)$ and constant density $\rho$. When the pressure variation is neglected, the extensional flow can serve as a useful approximation of the local free stream in certain low-Reynolds-number flows.

The corresponding fundamental solutions of the two-dimensional (plane-flow) problems can be derived in a similar way. For a two-dimensional Stokeslet

$$
\mathbf{f}_{S}=4 \pi \mu \alpha \delta(y) \delta(z)
$$


$\alpha$ being a two-dimensional vector in the $y, z$ plane, we have

$$
\begin{aligned}
& \mathrm{U}_{S}(\mathbf{x} ; \boldsymbol{\alpha})=\alpha \log r^{-1}+(\boldsymbol{\alpha} . \mathbf{x}) \mathbf{x} / r^{2} \quad\left(\mathbf{x}=(y, z), \quad r^{2}=y^{2}+z^{2}\right), \\
& P_{S}(\mathbf{x} ; \boldsymbol{\alpha})=2 \mu(\boldsymbol{\alpha} . \mathbf{x}) r^{-2}, \\
& \zeta_{S}(\mathbf{x} ; \boldsymbol{\alpha})=2(\boldsymbol{\alpha} \times \mathbf{x}) r^{-2} .
\end{aligned}
$$

The total force exerted on the fluid per unit breadth (in the $x$ direction) by a two-dimensional Stokeslet is

$$
\mathbf{F}=\int_{v_{0}} \mathbf{f}_{S} d V=4 \pi \mu \boldsymbol{\alpha}
$$

where $V_{c}$ is a control volume containing the Stokeslet.

Similarly, the other fundamental solutions for external flows can be readily converted from the three-dimensional to the two-dimensional form by simply replacing $R^{-1}$ by $\log (1 / r), R^{-n}(n \geqslant 2)$ by $r^{-n+1}$, and $4 \pi$ in the coefficients by $2 \pi$. The two-dimensional Stokeson has the same dependence on $\mathbf{x}$ and $r$ as a threedimensional one has on $\mathrm{x}$ and $R$ as shown in (13), except that the factor 2 in (13a) must be replaced by $\frac{3}{2}$ and the factor 10 in $(13 b)$ and the factor 5 in $(13 c)$ both by 4 . The two-dimensional versions of (15) and (16) are exactly the same expressions but with $\alpha, \beta$ and $\gamma$ designating two-dimensional vectors.

We now proceed to demonstrate the singularity method by presenting exact solutions to a number of Stokes-flow problems. Some of these have been previously obtained by different methods, the others are believed to be new.

\section{Uniform flow past a prolate spheroid}

We first consider the Stokes flow for a uniform free stream past a prolate spheroid

$$
x^{2} / a^{2}+r^{2} / b^{2}=1 \quad\left(r^{2}=y^{2}+z^{2}, \quad a \geqslant b\right),
$$

where the focal length $2 c$ and eccentricity $e$ are, as usual, related by

$$
c=\left(a^{2}-b^{2}\right)^{\frac{1}{2}}=e a \quad(0 \leqslant e<1) .
$$

With no loss of generality, the free-stream velocity may be taken as

$$
\mathbf{U}=U_{1} \mathbf{e}_{x}+U_{2} \mathbf{e}_{y},
$$

$\mathbf{e}_{x}, \mathbf{e}_{y}$ and $\mathbf{e}_{z}$ being the base vectors. This problem is chosen as a primary example since the body geometry is among the simplest exhibiting the effects of arbitrary body slenderness in low-Reynolds-number flow theory, and since its exact solution by the singularity method is relatively simple.

Partly guided by the known solution for potential flow past a prolate spheroid based on the singularity method (Chwang \& Wu $1974 b$ ), we try to construct the requisite solution by employing a line distribution of Stokeslets and potential doublets between the foci $x=-c$ and $c$ given by

$$
\begin{aligned}
\mathbf{u}=U_{1} \mathbf{e}_{x}+ & U_{2} \mathbf{e}_{y}-\int_{-c}^{c}\left[\alpha_{1} \mathbf{U}_{S}\left(\mathbf{x}-\xi ; \mathbf{e}_{x}\right)+\alpha_{2} \mathbf{U}_{S}\left(\mathbf{x}-\xi ; \mathbf{e}_{y}\right)\right] d \xi \\
& +\int_{-c}^{c}\left(c^{2}-\xi^{2}\right)\left[\beta_{1} \mathbf{U}_{D}\left(\mathbf{x}-\xi ; \mathbf{e}_{x}\right)+\beta_{2} \mathbf{U}_{D}\left(\mathbf{x}-\xi ; \mathbf{e}_{y}\right)\right] d \xi \\
p & =-\int_{-c}^{c}\left[\alpha_{1} P_{S}\left(\mathbf{x}-\xi ; \mathbf{e}_{x}\right)+\alpha_{2} P_{S}\left(\mathbf{x}-\xi ; \mathbf{e}_{y}\right)\right] d \xi
\end{aligned}
$$


where, here and below, $\xi=\xi \mathbf{e}_{x}$. The first integral in (22a) represents a line distri ${ }^{-}$ bution of Stokeslets, of constant strength $\alpha_{1}$ and $\alpha_{2}$, oriented in the negative- $x$ and negative- $y$ directions, respectively. The second integral denotes a line distribution of doublets, each of parabolic density and pointing in the $x$ and $y$ directions respectively, their parabolic density being suggested by the corresponding potential-flow solution. Obviously, (22a) and (22b) satisfy the (homogeneous) Stokes equations $(1 a)$ and $(1 b)$ in the flow field, and also satisfy the boundary conditions on $u$ and $p$ at infinity. To verify the no-slip condition on the spheroid surface, we make use of the integrated form of $\mathbf{u}$, which can be written as

$$
\begin{aligned}
\mathbf{u}= & U_{1} \mathbf{e}_{x}+U_{2} \mathbf{e}_{y}-\left(2 \alpha_{1} \mathbf{e}_{x}+\alpha_{2} \mathbf{e}_{y}\right) B_{1,0}-\left(\alpha_{1} r \mathbf{e}_{r}+\alpha_{2} y \mathbf{e}_{x}\right)\left(\frac{1}{R_{2}}-\frac{1}{R_{1}}\right) \\
& +\left(\alpha_{1} r \mathbf{e}_{x}-\alpha_{2} y \mathbf{e}_{r}\right) r B_{3,0}+\nabla\left\{-2 \beta_{1} B_{1,1}+\beta_{2} y\left[\frac{x-c}{r^{2}} R_{1}-\frac{x+c}{r^{2}} R_{2}+B_{1,0}\right]\right\},
\end{aligned}
$$

where $\mathbf{e}_{r}=\left(y \mathbf{e}_{y}+z \mathbf{e}_{z}\right) / r$ is the unit radial vector in the $y, z$ plane and

$$
\begin{gathered}
R_{1}=\left[(x+c)^{2}+r^{2}\right]^{\frac{1}{2}}, \quad R_{2}=\left[(x-c)^{2}+r^{2}\right]^{\frac{1}{2}}, \\
B_{m, n}(\mathbf{x}) \equiv \int_{-c}^{c} \frac{\xi^{n} d \xi}{|\mathrm{x}-\xi|^{m}} \quad(n=0,1,2, \ldots ; m=-1,1,3,5, \ldots) \\
B_{1,0}=\log \frac{R_{2}-(x-c)}{R_{1}-(x+c)}, \quad B_{1,1}=R_{2}-R_{1}+x B_{1,0} \\
B_{3,0}=\frac{1}{r^{2}}\left(\frac{x+c}{R_{1}}-\frac{x-c}{R_{2}}\right), \quad B_{3,1}=\left(\frac{1}{R_{1}}-\frac{1}{R_{2}}\right)+x B_{3,0}
\end{gathered}
$$

As the function $B_{m, n}(\mathbf{x})$ appears frequently in the analysis, we give here its recurrence formula for future convenience:

$$
B_{m, n}=-\frac{c^{n-1}}{m-2}\left(\frac{1}{R_{2}^{m-2}}+\frac{(-1)^{n}}{R_{1}^{m-2}}\right)+\frac{n-1}{m-2} B_{m-2, n-2}+x B_{m, n-1} \quad(n \geqslant 2) .
$$

Now, on the spheroid surface (21), designated by $S$,

$$
\begin{aligned}
& r^{2}=\left(1-e^{2}\right)\left(a^{2}-x^{2}\right), \quad R_{1}=a+e x, \quad R_{2}=a-e x, \\
& B_{1,0}=\log \frac{1+e}{1-e} \equiv L_{e}, \quad B_{3,0}=\frac{2 e}{\left(1-e^{2}\right)\left(a^{2}-e^{2} x^{2}\right)} .
\end{aligned}
$$

Hence the surface velocity becomes, after some simple manipulation,

$$
\begin{aligned}
\mathbf{u}_{0}= & {\left[U_{1}+\frac{2 \alpha_{1}}{e}-2\left(\alpha_{1}+\beta_{1}\right) L_{e}\right] \mathbf{e}_{x}-\frac{2}{e}\left(\alpha_{1}-\frac{2 e^{2} \beta_{1}}{1-e^{2}}\right) \frac{b^{2} \mathbf{e}_{x}+e^{2} x r \mathbf{e}_{r}}{a^{2}-e^{2} x^{2}} } \\
& +\left[U_{2}-\frac{2 e \beta_{2}}{1-e^{2}}-\left(\alpha_{2}-\beta_{2}\right) L_{e}\right] \mathbf{e}_{y}-2 e\left(\alpha_{2}-\frac{2 e^{2} \beta_{2}}{1-e^{2}}\right) y \frac{b^{2} x \mathbf{e}_{x}+a^{2} r \mathbf{e}_{r}}{b^{2}\left(a^{2}-e^{2} x^{2}\right)}
\end{aligned}
$$

The no-slip condition on $S$ is clearly satisfied if

$$
\begin{aligned}
& \alpha_{1}=2 \beta_{1} e^{2} /\left(1-e^{2}\right)=U_{1} e^{2}\left[-2 e+\left(1+e^{2}\right) L_{e}\right]^{-1}, \\
& \alpha_{2}=2 \beta_{2} e^{2} /\left(1-e^{2}\right)=2 U_{2} e^{2}\left[2 e+\left(3 e^{2}-1\right) L_{e}\right]^{-1} .
\end{aligned}
$$

This completes the required solution. 
The force experienced by the spheroid, by superposition of (4), is

$$
\mathbf{F}=-8 \pi \mu \int_{-\varepsilon}^{c}\left(-\alpha_{1} \mathbf{e}_{x}-\alpha_{2} \mathbf{e}_{y}\right) d x \equiv 6 \pi \mu a\left[U_{1} C_{F 1} \mathbf{e}_{x}+U_{2} C_{F 2} \mathbf{e}_{y}\right]
$$

with the force coefficients given by

$$
\begin{aligned}
& C_{F^{\prime} 1}=\frac{8}{3} \frac{c \alpha_{1}}{a U_{1}}=\frac{8}{3} e^{3}\left[-2 e+\left(1+e^{2}\right) \log \frac{1+e}{1-e}\right]^{-1}, \\
& C_{F 2}=\frac{8}{3} \frac{c \alpha_{2}}{a U_{2}}=\frac{16}{3} e^{3}\left[2 e+\left(3 e^{2}-1\right) \log \frac{1+e}{1-e}\right]^{-1} .
\end{aligned}
$$

This result for the force is in agreement with that given by Oberbeck (1876) and Jeffery (1922). The present solution also facilitates the determination of other physical quantities, such as the pressure forces.

The pressure, upon carrying out the integration in $(22 b)$, is given by

$$
p=2 \mu \alpha_{1}\left(\frac{1}{R_{1}}-\frac{1}{R_{2}}\right)+2 \mu \alpha_{2} \frac{y}{r^{2}}\left(\frac{x-c}{R_{2}}-\frac{x+c}{R_{1}}\right),
$$

which assumes on the spheroid surface $S$ the value

$$
p(x, r(x))=-4 \mu e\left(\alpha_{1} x+\alpha_{2} \frac{a^{2}}{b^{2}} y\right) /\left(a^{2}-e^{2} x^{2}\right) .
$$

Noting that the unit outward normal $\mathbf{n}$ on $S$ is

$$
\mathbf{n}=\left(\frac{b}{a} x \mathbf{e}_{x}+\frac{a}{b} y \mathbf{e}_{y}+\frac{a}{b} z \mathbf{e}_{z}\right)\left(a^{2}-e^{2} x^{2}\right)^{-\frac{1}{2}}
$$

we find the pressure contribution to the total force to be

$$
\begin{gathered}
\mathbf{F}_{p}=-\int_{S} p \mathbf{n} d S=6 \pi \mu a\left[U_{1} C_{p 1} \mathbf{e}_{x}+U_{2} C_{p 2} \mathbf{e}_{y}\right], \\
C_{p 1}=\frac{1-e^{2}}{2 e^{3}}\left[-2 e+\log \frac{1+e}{1-e}\right] C_{F 1}, \\
C_{p 2}=\frac{1}{4 e^{3}}\left[2 e-\left(1-e^{2}\right) \log \frac{1+e}{1-e}\right] C_{F^{\prime} 2}
\end{gathered}
$$

This result gives the relative magnitudes $C_{p 1} / C_{F^{1} 1}$ and $C_{p 2} / C_{F^{2}}$ for prolate spheroids of arbitrary eccentricity $e$. The remaining contribution to the force is due to the viscous skin friction.

In the limiting case of a sphere (as $a \rightarrow b$, or $e \rightarrow 0$ ), (26) reduces to

$$
\mathbf{u}=\mathbf{U}-\frac{3 a}{4}\left[\frac{\mathbf{U}}{R}+\frac{(\mathbf{U} \cdot \mathbf{x}) \mathbf{x}}{R^{3}}\right]+\frac{a^{3}}{4} \nabla\left(\nabla \cdot \frac{\mathbf{U}}{R}\right)
$$

and by (28) and (31),

$$
C_{F 1}=C_{F 2}=1, \quad C_{p 1} / C_{F^{1} 1}=C_{p 2} / C_{F 2}=\frac{1}{3},
$$

all being well-known results. 


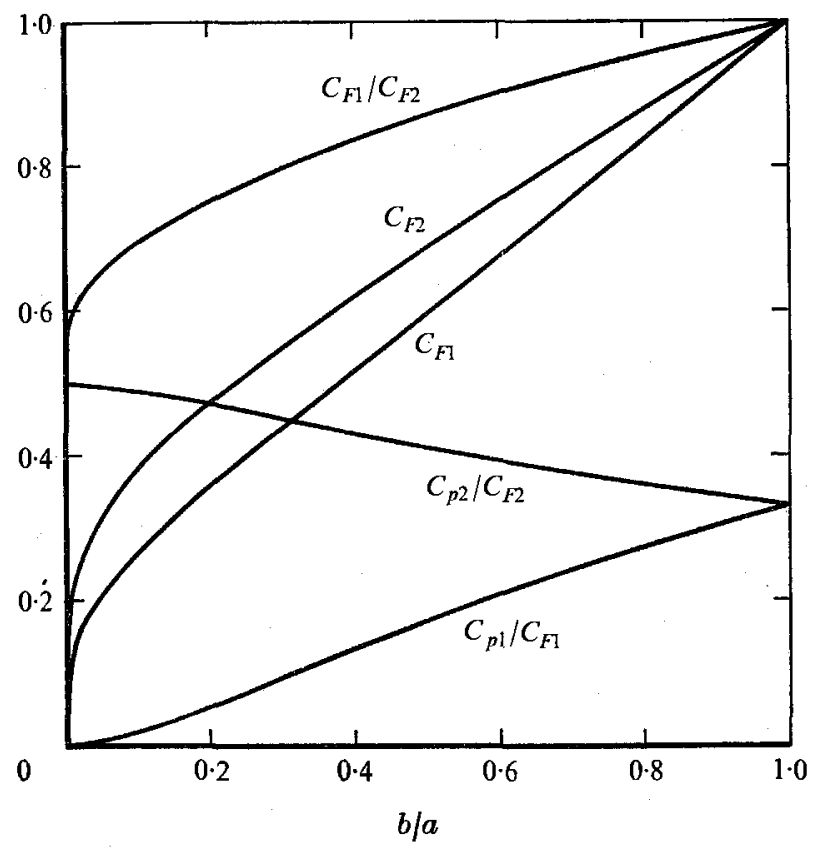

Figure 1. The longitudinal and transverse force coefficients $C_{F 1}$ and $C_{F 2}\left[C_{F i}=F_{i} / 6 \pi \mu U_{i} a\right.$, $i=1,2$, see (28)] of a prolate spheroid of axis ratio $b / a$ immersed in a Stokes flow with a uniform free stream. $C_{p 1}$ and $C_{p 2}$ are the pressure contributions to the respective force coefficients.

In the other extreme of very slender spheroids, or for a slenderness parameter

$$
\epsilon \equiv b / a=\left(1-e^{2}\right)^{\frac{1}{2}} \ll 1,
$$

the force coefficients have the following asymptotic behaviour:

$$
C_{F 1} \simeq \frac{2}{3} \frac{1}{\log (2 a / b)-\frac{1}{2}}\left[1+O\left(\epsilon^{2}\right)\right], \quad C_{F 2} \simeq \frac{4}{3} \frac{1}{\log (2 a / b)+\frac{1}{2}}\left[1+O\left(\epsilon^{2}\right)\right],
$$

which agrees with Tillett's (1970) result based on slender-body theory. Further, from the expansion

$$
C_{F 1} / C_{F 2} \simeq \frac{1}{2}\left\{1+[\log (2 / \epsilon)]^{-1}+O(\log \epsilon)^{-2}\right\}
$$

we note that the ratio $C_{F 1} / C_{F 2}$ tends to $\frac{1}{2}$ as $\epsilon \rightarrow 0$, but that this asymptotic limit is approached logarithmically since $d\left(C_{F^{1}} / C_{F^{2}}\right) / d \epsilon \simeq \frac{1}{2}\left[\epsilon \log ^{2}(2 / \epsilon)\right]^{-1}$, which is unbounded as $\epsilon \rightarrow 0$. The corresponding $C_{p 1}$ and $C_{p 2}$ are

$$
C_{p 1} \simeq \epsilon^{2}[\log (2 / \epsilon)-1] C_{F 1}, \quad C_{p 2} \simeq \frac{1}{2}\left[1-\epsilon^{2} \log (2 / \epsilon)\right] C_{F 2},
$$

indicating that $C_{p 1}$ becomes insignificant for the longitudinal translation of an elongated spheroid, whereas $C_{p 2}$ is nearly $\frac{1}{2} C_{F 2}$ and is rather insensitive to variations in $\epsilon$ when $\epsilon$ is small. These salient features of the force coefficients are clearly exhibited in figure 1, which is a plot of $C_{F 1}, C_{F 2}, C_{p 1}$ and $C_{p 2}$ over the range $0 \leqslant b / a \leqslant 1$.

Other important quantities are the local cross-sectional force coefficients since 
they form the basis of slender-body theory. The local force acting on a unit cross-section of a spheroid at a station $x$ can be determined from

$$
\mathbf{F}_{l}(x)=\int_{\mathscr{C}}(-p \mathbf{n}+\boldsymbol{\tau n}) \frac{r(x)}{\mathbf{n} \cdot \mathbf{e}_{r}} d \theta \equiv U_{1} C_{s}(x) \mathbf{e}_{x}+U_{2} C_{n}(x) \mathbf{e}_{y},
$$

where $\mathscr{C}$ is the contour around $r=r(x)$ and $\theta$ is the cylindrical polar angle. We remark that $d S=r(x) d \theta d x /\left(\mathbf{n} . \mathbf{e}_{r}\right)$ is a surface element of the spheroid, so that the differential force $F_{l}(x)$ and the total force $F$ [see $(28 a)$ ] are related by

$$
\mathbf{F}=\int_{-a}^{a} \mathbf{F}_{l}(x) d x .
$$

The above definition of $C_{s}(x)$ and $C_{n}(x)$ is conventional in the literature of slenderbody theory (see Gray \& Hancock 1955). By making use of the present exact solution (26) and (29), the line integral in (35a) can be evaluated, which is not tedious, and we find that

$$
\begin{aligned}
\int_{\mathscr{C}}-p \mathbf{n} \frac{r(x)}{\mathbf{n} \cdot \mathbf{e}_{r}} d \theta & =\frac{4 \pi \mu e}{a^{2}-e^{2} x^{2}}\left\{2 \mathbf{e}_{x}\left(1-e^{2}\right) \alpha_{1} x^{2}+\mathbf{e}_{y} \alpha_{2}\left(a^{2}-x^{2}\right)\right\}, \\
\int_{\mathscr{C}} \tau \mathbf{n} \frac{r(x)}{\mathbf{n} \cdot \mathbf{e}_{r}} d \theta & =\frac{4 \pi \mu e}{a^{2}-e^{2} x^{2}}\left\{2 \mathbf{e}_{x} \alpha_{1}\left(a^{2}-x^{2}\right)+\mathbf{e}_{y} \alpha_{2}\left[2\left(1-e^{2}\right) x^{2}+a^{2}-x^{2}\right]\right\},
\end{aligned}
$$

from which it follows that

$$
C_{s}(x)=8 \pi \mu e \alpha_{1} / U_{1}, \quad C_{n}(x)=8 \pi \mu e \alpha_{2} / U_{2} \quad(-a \leqslant x \leqslant a),
$$

where $\alpha_{1}$ and $\alpha_{2}$ are given by (27). This result, which would hardly be expected intuitively, shows that the cross-sectional force coefficients $C_{s}$ and $C_{n}$ are both constant, independent of $x$, for a prolate spheroid of arbitrary eccentricity, including the sphere! Further, by (28) and (36a),

$$
C_{s}=3 \pi \mu C_{F 1}, \quad C_{n}=3 \pi \mu C_{F 2} ;
$$

the values of $C_{s} / C_{n}$ for different eccentricities $e$ can therefore be read from the curve of $C_{F 1} / C_{F 2}$ in figure 1 . For very slender spheroids, it follows from (34) and (36b) that

$$
C_{s}=\frac{2 \pi \mu}{\log (2 a / b)-\frac{1}{2}}, \quad C_{n}=\frac{4 \pi \mu}{\log (2 a / b)+\frac{1}{2}} \quad(b / a \ll 1) .
$$

This result should be compared with slender-body theory, which provides, for an elongated rod of radius $b$ and length $2 a$, the formula

$$
C_{s}=\frac{2 \pi \mu}{\log (2 a / b)-\frac{1}{2}}, \quad C_{n}=\gamma C_{s}
$$

$\gamma$ being a constant. According to Gray \& Hancock (1955), $\gamma=2$, whereas Cox (1970) has recommended that $\gamma$ be the ratio $C_{n} / C_{s}$ determined by using (37), which is a function of $a / b$, and is always less than 2 .

Regarding the structure of the present solution we further note that only Stokeslets and doublets are required for a prolate spheroid in translation and their line distributions all terminate at the two foci. The distance between one 

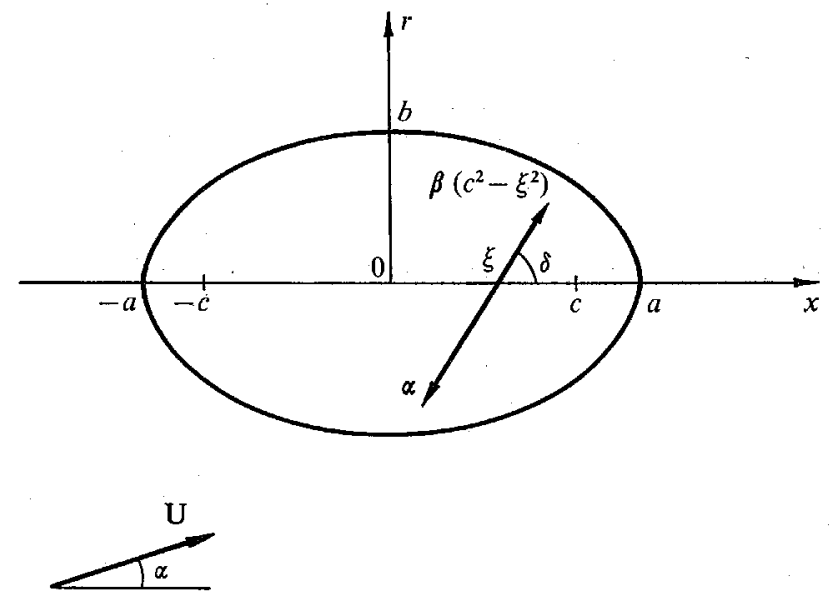

Figure 2. The orientations of the distributed Stokeslets and doublets for uniform flow past a prolate spheroid.

focus and the nearer end of the body is related to the radius of curvature $\widetilde{R}_{a}$ at the nose by

$$
\widetilde{R}_{a}=(1+e)(a-c) .
$$

For elongated spheroids, this becomes $a-c=\frac{1}{2} \widetilde{R}_{a}$ as $e \rightarrow 1$. This asymptotic end property has been generally recognized for potential flows (Landweber 1951) and has also been conjectured for the low-Reynolds-number case (Tuck 1970; Tillett 1970).

Another point of interest is that, since $\alpha_{1} / \alpha_{2}=\beta_{1} / \beta_{2}$ by $(27 a, b)$, the resultant Stokeslet and the resultant doublet subtend the same angle

$$
\delta=\tan ^{-1}\left(\alpha_{2} / \alpha_{1}\right)=\tan ^{-1}\left(\beta_{2} / \beta_{1}\right)
$$

at the $x$ axis. This angle is related, in virtue of $(28 b, c)$, to the incidence angle $\alpha=\tan ^{-1}\left(U_{2} / U_{1}\right)$ by (see figure 2 )

$$
\tan \delta=\alpha_{2} / \alpha_{1}=\left(C_{F 2} / C_{F 1}\right) \tan \alpha .
$$

For elongated spheroids this relationship becomes, according to (34b),

$$
\tan \delta \simeq 2 \tan \alpha+O(\log \epsilon)^{-1} .
$$

In the same limit, however, the doublets $\beta_{1}$ and $\beta_{2}$ become increasingly weaker than the Stokeslets, since by $(27 a, b)$,

$$
\beta_{1} \simeq \frac{1}{2} \epsilon^{2} \alpha_{1}, \quad \beta_{2} \simeq \frac{1}{2} \epsilon^{2} \alpha_{2} \quad(\epsilon=b / a \ll 1) .
$$

The approximation of the solution by leaving doublets out when evaluating Stokes flows involving slender bodies has been practised before in the literature. While this is valid for elongated spheroids, to attach a general validity to this approximation, for arbitrary slender bodies, may be premature. A counter-example is the twodimensional circular cylinder (its effective slenderness parameter being $\epsilon=0$ ), for which a doublet is still required for constructing the near-field solution. Another example appears in §14. 
Finally, it is of importance to estimate the range of validity of the present solution by assessing the magnitude of the neglected inertial forces relative to the terms retained. It is convenient to separate the longitudinal and transverse motions and to evaluate the flow quantities on a spheroidal control surface, which is taken to be confocal with the body surface, namely on $r=r_{*}(x)$, where

$$
r_{*}^{2}=\left(1-e_{*}^{2}\right)\left(a_{*}^{2}-x^{2}\right) \quad\left(e_{*} a_{*}=e a, e_{*}<e\right) .
$$

For the longitudinal motion (with $U_{2}=0$ ), a typical leading term of the inertial force is $u \partial \mathbf{u} / \partial x$, while for the transverse motion such a term is $v \partial \mathbf{u} / \partial y$, where $\mathbf{u}=(u, v, w)$. The estimate of the viscous force may be taken as $\left|\mu \nabla^{2} \mathbf{u}\right|$ or $|\nabla p|$ since they are equal according to the Stokes approximation. We find the ratio of the local inertial effects to the local viscous effects (evaluated at $r=r_{*}$ ) to be

$$
\begin{array}{ll}
\frac{|\rho u \partial \mathbf{u} / \partial x|}{|\nabla p|} \simeq O\left(\frac{u r_{*}}{\nu}\right) & \text { (longitudinal motion), } \\
\frac{|\rho v \partial \mathbf{u} / \partial y|}{|\nabla p|} \simeq O\left(\frac{v r_{*}}{\nu}\right) \quad \text { (transverse motion), }
\end{array}
$$

where $v=\mu / \rho$. Since $u$ and $v$ tend to zero as the body surface is approached and $u \rightarrow U_{1}$ and $v \rightarrow U_{2}$ at infinity, the above ratios will remain small in a neighbourhood of the body if

$$
R_{b}<R_{a} \ll 1 \quad\left(R_{a}=U a / v, R_{b}=U b / v, U=|\mathbf{U}|\right),
$$

no matter how small the parameter $\epsilon=b / a=R_{b} / R_{a}$ is. Under this condition, the justification for the present results (for the force at least) as a valid first approximation for a small longitudinal Reynolds number $R_{a}$ is entirely parallel to the classical argument for the sphere. However, the situation becomes very different when

$$
R_{b} \ll 1, \quad R_{a} \text { not necessarily small. }
$$

In this case the 'local' Reynolds number in (43) may be recast as

$$
U r_{*} / \nu=\left(r_{*} / a\right) R_{a}
$$

If this ratio is required to be small in order to justify the Stokes approximation, $r_{*} / a$ may be very much restricted by this requirement, especially when $R_{a} \gg 1$. In fact, the upper bound on $r_{*} / a$ could be so small that the local velocity $\mathbf{u}_{*}$ would still deviate appreciably from the free-stream value U. Under such circumstances the present solution would no doubt break down. This class of problems will be discussed in a future paper.

\section{Longitudinal shear flow past a prolate spheroid}

We next consider the shear flow $\mathbf{U}=\Omega_{3} y \mathbf{e}_{x}$ past a prolate spheroid specified by (21). The fact that the $y$ derivative of $U$ is a constant vector suggests, in view of the previous example, that the required singularities include a line distribution of Stokes doublets associated with the base vectors $\mathbf{e}_{x}$ and $\mathbf{e}_{y}$, which can be assembled more conveniently in terms of a stresslet, a rotlet (whose direction is 
opposite to the vorticity of the primary flow), and a potential quadrupole (which is generally associated with a stresslet much like a doublet is associated with a Stokeslet). On this basis we find the solution to be of the form

$$
\begin{array}{r}
\mathbf{u}=\Omega_{3} y \mathbf{e}_{x}+\int_{-c}^{c}\left(c^{2}-\xi^{2}\right)\left[\alpha_{3} \mathbf{U}_{S S}\left(\mathbf{x}-\xi ; \mathbf{e}_{x}, \mathbf{e}_{y}\right)+\gamma_{3} \mathbf{U}_{R}\left(\mathbf{x}-\xi ; \mathbf{e}_{z}\right)\right] d \xi \\
\quad+\beta_{3} \int_{-c}^{c}\left(c^{2}-\xi^{2}\right)^{2} \frac{\partial}{\partial y} \mathbf{U}_{D}\left(\mathbf{x}-\xi ; \mathbf{e}_{x}\right) d \xi, \\
p=\alpha_{3} \int_{-c}^{c}\left(c^{2}-\xi^{2}\right) P_{S S}\left(\mathbf{x}-\xi ; \mathbf{e}_{x}, \mathbf{e}_{y}\right) d \xi
\end{array}
$$

where $\alpha_{3}, \beta_{3}$ and $\gamma_{3}$ are constants and the fundamental solutions $\mathbf{U}_{S S}, \mathbf{U}_{R}$, etc. are given in $\S 2$. These integrals can all be expressed in terms of the function $B_{m, n}(\mathbf{x})$ defined by (24); the final result reads

$$
\begin{aligned}
\mathbf{u}=\Omega_{3} y \mathbf{e}_{x}+\alpha_{3} y\left[\left(2 A_{1}+A_{3}\right) \mathbf{e}_{x}+2 r \mathbf{e}_{r} B_{3,1}\right] \\
+\gamma_{3}\left[2 \mathbf{e}_{y} B_{1,1}-\mathbf{e}_{x} y A_{3}\right]+4 \beta_{3} \nabla\left[y\left(c^{2} B_{3,1}-B_{3,3}\right)\right],
\end{aligned}
$$

where

$$
A_{1}=x B_{3,1}-B_{3,2}, \quad A_{3}=c^{2} B_{3,0}-B_{3,2} \text {. }
$$

From this result we find, after some calculation, that the no-slip condition $(\mathbf{u}=0$ on $S)$ is satisfied if

$$
\begin{aligned}
& \alpha_{3}=\frac{4 e^{2}}{1-e^{2}} \beta_{3}=2 e^{2} \gamma_{3}\left[-2 e+\log \frac{1+e}{1-e}\right]\left[2 e\left(2 e^{2}-3\right)+3\left(1-e^{2}\right) \log \frac{1+e}{1-e}\right]^{-1}, \\
& \gamma_{3}=\Omega_{3}\left(1-e^{2}\right)\left[-2 e+\left(1+e^{2}\right) \log \frac{1+e}{1-e}\right]^{-1} .
\end{aligned}
$$

The net moment on the spheroid is contributed by the rotlet only; it can be determined by superposition of (9), giving

$$
\mathbf{M}_{12}=-8 \pi \mu \int_{-c}^{c} \gamma_{3}\left(c^{2}-\xi^{2}\right) \mathbf{e}_{z} d \xi=-\frac{32}{3} \pi \mu c^{3} \gamma_{3} \mathbf{e}_{z}
$$

or in coefficient form,

$$
C_{M 12}=\frac{M_{12}}{8 \pi \mu a b^{2} \Omega_{3}}=\frac{4}{3} \frac{e^{3}}{1-e^{2}} \frac{\gamma_{3}}{\Omega_{3}}=\frac{4}{3} e^{3}\left[-2 e+\left(1+e^{2}\right) \log \frac{1+e}{1-e}\right]^{-1} .
$$

The resultant moment acts on the spheroid in the direction of the vorticity vector of the primary flow. There is no net force on a spheroid immersed in this shear flow since the solution contains no Stokeslet; this is because the shear flow is centred about the longitudinal axis of the spheroid.

In the limiting case of a sphere $(a \rightarrow b$, or $e \rightarrow 0)$, we deduce from the above result that, as $e \rightarrow 0$,

$$
\begin{gathered}
\alpha_{3} \int_{-c}^{c}\left(c^{2}-\xi^{2}\right) d \xi=-\frac{5}{6} \Omega a^{3}, \quad \gamma_{3} \int_{-c}^{c}\left(c^{2}-\xi^{2}\right) d \xi=\frac{1}{2} \Omega a^{3}, \\
\beta_{3} \int_{-c}^{c}\left(c^{2}-\xi^{2}\right)^{2} d \xi=-\frac{1}{6} \Omega a^{5}
\end{gathered}
$$


where the subscripts on $\Omega$ have been omitted. Consequently, the corresponding limiting values of $\mathbf{u}$ and $\mathbf{M}$ are

$$
\begin{aligned}
\mathbf{u} & =\Omega\left[y \mathbf{e}_{x}-\frac{5}{6} a^{3} \frac{3 x y \mathbf{x}}{R^{5}}+\frac{a^{3}}{2} \frac{\mathbf{e}_{\varepsilon} \times \mathbf{x}}{R^{3}}-\frac{a^{5}}{6} \nabla \frac{\partial^{2}}{\partial x \partial y} \frac{1}{R}\right], \\
\mathbf{M} & =-4 \pi \mu \Omega a^{3} \mathbf{e}_{z} .
\end{aligned}
$$

This result agrees with the known solution of Burgers (1938) for a sphere in a shear flow. The present solution for the spheroid also agrees with that in Jeffery's (1922) general treatment of ellipsoids based on an analysis involving ellipsoidal harmonics. This problem has been further discussed recently by Cox (1971) using the singularity method, but only approximately for the case of a small shear rate in the free stream.

\section{Cross-flow with a longitudinal rate of shear past a prolate spheroid}

In the case of a free stream $\mathbf{U}=\Omega_{3}^{\prime} x \mathbf{e}_{y}$ flowing past the same spheroid as before, the structure of the solution is entirely analogous to the preceding case, namely

$$
\begin{array}{r}
\mathbf{u}=\Omega_{3}^{\prime} x \mathbf{e}_{y}-\int_{-c}^{c}\left(c^{2}-\xi^{2}\right)\left[\alpha_{3}^{\prime} \mathbf{U}_{S S}\left(\mathbf{x}-\xi ; \mathbf{e}_{x}, \mathbf{e}_{y}\right)+\gamma_{3}^{\prime} \mathbf{U}_{R}\left(\mathbf{x}-\xi ; \mathbf{e}_{z}\right)\right] d \xi \\
\quad-\beta_{3}^{\prime} \int_{-c}^{c}\left(c^{2}-\xi^{2}\right)^{2} \frac{\partial}{\partial x} \mathbf{U}_{D}\left(\mathbf{x}-\xi ; \mathbf{e}_{y}\right) d \xi \\
p=-\alpha_{3}^{\prime} \int_{-c}^{c}\left(c^{2}-\xi^{2}\right) P_{S S}\left(\mathbf{x}-\xi ; \mathbf{e}_{x}, \mathbf{e}_{y}\right) d \xi
\end{array}
$$

In fact, since $\partial \mathbf{U}_{D}\left(\mathbf{x} ; \mathbf{e}_{y}\right) / \partial x=\partial \mathbf{U}_{D}\left(\mathbf{x} ; \mathbf{e}_{x}\right) / \partial y$, the perturbation flow field has exactly the same functional expression as in (45). The three new coefficients $\alpha_{3}^{\prime}, \beta_{3}^{\prime}$ and $\gamma_{3}^{\prime}$ can be determined upon invoking the no-slip condition $\mathbf{u}=0$ on the body surface $S$ specified by (21), which gives

$$
\begin{gathered}
\alpha_{3}^{\prime}=\frac{4 e^{2}}{1-e^{2}} \beta_{3}^{\prime}=e^{2} \gamma_{3}^{\prime}\left[-2 e+\left(1-e^{2}\right) \log \frac{1+e}{1-e}\right]\left[2 e\left(2 e^{2}-3\right)+3\left(1-e^{2}\right) \log \frac{1+e}{1-e}\right]^{-1}, \\
\gamma_{3} / \Omega_{3}=\left(1-e^{2}\right) \gamma_{3}^{\prime} / \Omega_{3}^{\prime},
\end{gathered}
$$

$\gamma_{3}$ being given by (47). The corresponding moment on the spheroid is

or

$$
\begin{gathered}
\mathbf{M}_{21}=-8 \pi \mu \int_{-c}^{c}\left[-\gamma_{3}^{\prime}\left(c^{2}-\xi^{2}\right) \mathbf{e}_{z}\right] d \xi=\frac{32}{3} \pi \mu c^{3} \gamma_{3}^{\prime} \mathbf{e}_{z}, \\
C_{M 21}=\frac{M_{21}}{8 \pi \mu a b^{2} \Omega_{3}^{\prime}}=\frac{4}{3} \frac{e^{3}}{1-e^{2}} \frac{\gamma_{3}^{\prime}}{\Omega_{3}^{\prime}}=\frac{1}{1-e^{2}} C_{M 12}
\end{gathered}
$$

on account of (52b) and (48b). We may note that, as a rule, the moment exerted by a shear flow on an axisymmetric body acts in the direction of the vorticity vector of the primary flow. Through this rule, the present result covers all the cross-flows having a longitudinal rate of shear. 
If the uniform shear flows of this section and $\$ 4$ are superimposed, the resultant moment (by superposition of (48) and (53)) will vanish when $\gamma_{3}=\gamma_{3}^{\prime}$, or after making use of $(52 b)$, when

$$
\Omega_{3} / \Omega_{3}^{\prime}=a^{2} / b^{2} .
$$

Therefore, a spheroid experiences no moment when the vorticity vectors of the longitudinal and transverse flows are in opposite directions and have the ratio of their magnitudes equal to the square of the axis ratio $r_{e}=a / b$.

\section{Cross-flow with a transverse rate of shear past a prolate spheroid}

In this case a prolate spheroid is held fixed in a flow which is in the $y$ direction and sheared in the $z$ direction with, for example, the free stream $\mathrm{U}=\Omega_{1} z \mathbf{e}_{y}$. Again, on the basis of the symmetry properties relating the singularities to the primary flow as explained in the previous two cases, the solution can be expressed in the form

$$
\begin{array}{r}
\mathbf{u}=\Omega_{1} z \mathbf{e}_{y}+\int_{-c}^{c}\left(c^{2}-\xi^{2}\right)\left[\alpha_{4} \mathbf{U}_{S S}\left(\mathbf{x}-\xi ; \mathbf{e}_{y}, \mathbf{e}_{z}\right)+\gamma_{4} \mathbf{U}_{R}\left(\mathbf{x}-\xi ; \mathbf{e}_{x}\right)\right] d \xi \\
\quad+\beta_{4} \int_{-o}^{c}\left(c^{2}-\xi^{2}\right)^{2} \frac{\partial}{\partial z} \mathbf{U}_{D}\left(\mathbf{x}-\xi ; \mathbf{e}_{y}\right) d \xi \\
p=\alpha_{4} \int_{-c}^{c}\left(c^{2}-\xi^{2}\right) P_{S S}\left(\mathbf{x}-\xi ; \mathbf{e}_{y}, \mathbf{e}_{z}\right) d \xi
\end{array}
$$

These integrals can all be expressed in terms of the function $B_{m, n}(\mathbf{x})$ defined by (24e), whose value at the spheroid surface can be evaluated straightforwardly with the aid of (25). Curtailing the details, we find that the no-slip condition at the body surface is satisfied by $(55 a)$ if

$$
\begin{aligned}
& \alpha_{4}=\frac{4 e^{2}}{1-e^{2}} \beta_{4}=2 \Omega_{1} e^{2}\left(1-e^{2}\right)\left[2 e\left(3-5 e^{2}\right)-3\left(1-e^{2}\right)^{2} \log \frac{1+e}{1-e}\right]^{-1}, \\
& \gamma_{4}=\frac{1}{2} \Omega_{1}\left(1-e^{2}\right)\left[2 e-\left(1-e^{2}\right) \log \frac{1+e}{1-e}\right]^{-1} .
\end{aligned}
$$

The resultant moment is obtained by integrating the rotlet distribution, giving

or

$$
\begin{gathered}
\mathbf{M}_{23}=-8 \pi \mu \int_{-c}^{c} \gamma_{4}\left(c^{2}-\xi^{2}\right) \mathbf{e}_{x} d \xi=-\frac{32}{3} \pi \mu c^{3} \gamma_{4} \mathbf{e}_{x} \\
C_{M 23}=\frac{M_{23}}{8 \pi \mu a b^{2} \Omega_{1}}=\frac{4}{3} \frac{e^{3}}{1-e^{2}} \frac{\gamma_{4}}{\Omega_{1}}=\frac{2}{3} e^{3}\left[2 e-\left(1-e^{2}\right) \log \frac{1+e}{1-e}\right]^{-1} .
\end{gathered}
$$

If the spheroid is released in this particular shear flow, the applied moment will clearly make the spheroid rotate about its longitudinal axis until it reaches a steady angular velocity, say $-\Omega_{0} \mathbf{e}_{x}$. But if this steady rotation took place in a viscous fluid otherwise at rest, it would exert a moment on the fluid given by (see equations (43) and (44) of part 1)

$$
\mathbf{M}_{0}=\frac{32}{3} \pi \mu c^{3} \Omega_{0}\left(1-e^{2}\right)\left[2 e-\left(1-e^{2}\right) \log \frac{1+e}{1-e}\right]^{-1} \mathbf{e}_{x}
$$




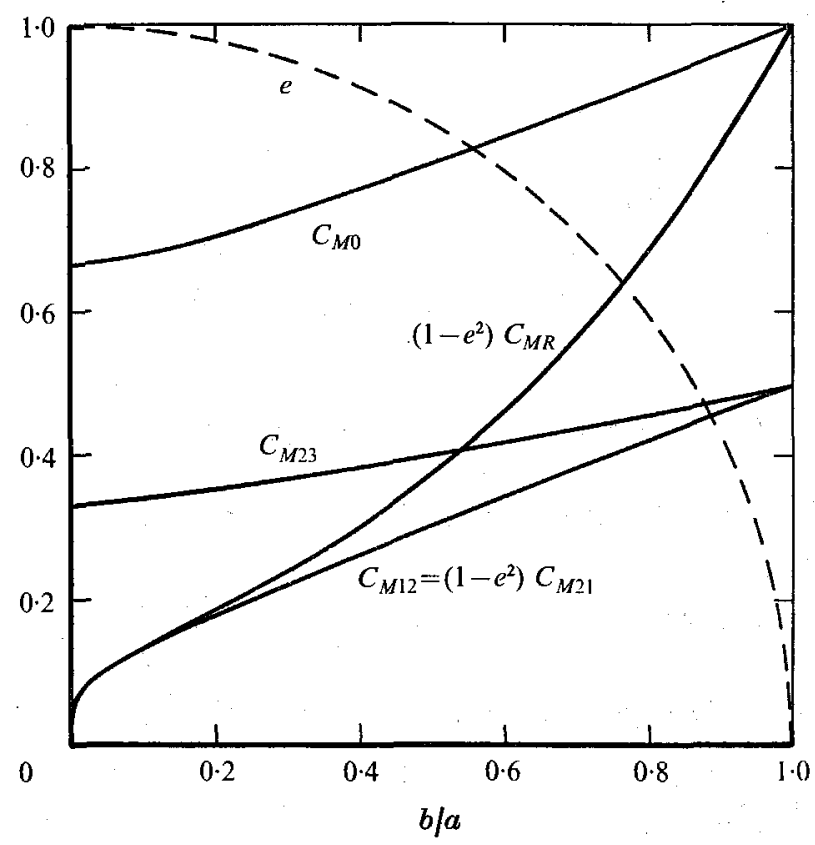

FIGURE 3. Various moment coefficients of a prolate spheroid of eccentricity $e$ and axis ratio $b / a=\left(1-e^{2}\right)^{\frac{1}{1}} . C_{M 12}=M_{12} / 8 \pi \mu \Omega_{3} a b^{2}$ is the moment coefficient due to a longitudinal shear flow with shear rate $\Omega_{3}, C_{M 21}$ that due to a cross-flow with longitudinal shear, $C_{M 23}$ that due to a cross-flow with transverse shear, $C_{M R}$ that due to rotation about a minor axis and $C_{M 0}$ that due to rotation about the major axis.

or using the coefficient form and comparing it with (57),

$$
C_{M 0}=M_{0} / 8 \pi \mu a b^{2} \Omega_{0}=2 C_{M 23} \text {. }
$$

Since the action and reaction must balance for a free spheroid rotating in a steady state, we must have, in view of the linearity of Stokes flows, $\mathbf{M}_{23}+\mathbf{M}_{0}=0$, from which it follows that

$$
\Omega_{0}=\frac{1}{2} \Omega_{1} \text {, }
$$

which states that the steady angular velocity $\Omega_{0}$ of a freely rotating spheroid is half the basic flow vorticity, as might be expected.

The four moment coefficients $C_{M 12}$ of (48), $C_{M 21}$ of (53), $C_{M 23}$ of (57) and $C_{M 0}$ of $(58 b)$ are shown in figure 3 over the range $0 \leqslant b / a \leqslant 1$. These results are valid if $\Omega a^{2} / \nu \ll 1$ for the first two cases and if $\Omega b^{2} / \nu \ll 1$ for the third, while the last has already been discussed in part 1 .

\section{Rotation of a prolate spheroid about a minor axis}

The problem of the rotation of a prolate spheroid about a minor axis, with angular velocity $\boldsymbol{\Omega}=\Omega \mathbf{e}_{z}$ say, can be readily resolved by appropriate superposition of the solutions of the preceding shear-flow problems. This possibility is realized upon observing that the flow velocity relative to the body has at large 
distances the form $\mathbf{U}=(\Omega y,-\Omega x, 0)$, each component being a shear flow already discussed. In drawing this analogy we note that the inertial effect arising from the transformation from an absolute frame to the body frame may be neglected, together with other inertia forces, in accordance with the basic assumption for low-Reynolds-number flow, which here requires that $\Omega a^{2} / \nu \ll 1$. Therefore, the solution, expressed in the body frame, can be written as

$$
\begin{aligned}
& \mathbf{u}=\Omega y \mathbf{e}_{x}-\Omega x \mathbf{e}_{y}+\mathbf{u}_{1}(\mathbf{x})+\mathbf{u}_{2}(\mathbf{x}), \\
& p=p_{1}(\mathbf{x})+p_{2}(\mathbf{x}),
\end{aligned}
$$

where $\left(\mathbf{u}_{1}, p_{1}\right)$ and $\left(\mathrm{u}_{2}, p_{2}\right)$ are given by (45) and (51), respectively, with $\Omega_{3}=\Omega$ and $\Omega_{3}^{\prime}=-\Omega$.

Consequently, the resultant moment on the rotating spheroid, by superposition of $(48 a)$ and $(53 a)$, is

$$
\mathbf{M}_{R}=-\frac{32}{3} \pi \mu c^{3}\left(\gamma_{3}-\gamma_{3}^{\prime}\right) \mathbf{e}_{z}=-\frac{32}{3} \pi \mu c^{3}\left(\frac{2-e^{2}}{1-e^{2}}\right) \gamma_{3} \mathbf{e}_{z},
$$

where $\gamma_{3}$ is given by (47b) (with $\Omega_{3}=\Omega$ ), or in coefficient form,

$$
C_{M R}=\frac{M_{R}}{8 \pi \mu a b^{2} \Omega}=\frac{4}{3} e^{3}\left(\frac{2-e^{2}}{1-e^{2}}\right)\left[-2 e+\left(1+e^{2}\right) \log \frac{1+e}{1-e}\right]^{-1},
$$

which agrees with the result obtained by Gans (1928). The moment coefficient $C_{M R}$ is also shown in figure 3 for comparison with the other cases.

\section{Extensional flow past a prolate spheroid}

The simplest case of an extensional flow [also called 'hyperbolic flow', see $(\mathbf{1 7 b})]$ past the present spheroid is the one with an axisymmetric free stream

$$
\mathrm{U}=2 \Omega x \mathbf{e}_{x}-\Omega y \mathbf{e}_{y}-\Omega z \mathbf{e}_{z} .
$$

It corresponds to a radial inflow and a longitudinal outflow. From the axial symmetry it is obvious that no rotlet is required for constructing the solution. In fact, the form of the gradient of $\mathbf{U}$ suggests that we adopt stresslets and potential quadrupoles in a diagonal combination of the form

$$
\begin{gathered}
\mathbf{u}=\mathbf{U}+\mathbf{u}_{s s}(\mathbf{x})+\mathbf{u}_{p q}(\mathbf{x}), \\
\mathbf{u}_{s s}=\frac{1}{3} \alpha_{5} \int_{-c}^{c}\left(c^{2}-\xi^{2}\right)\left[2 \mathbf{U}_{S S}\left(\mathbf{x}-\xi ; \mathbf{e}_{x}, \mathbf{e}_{x}\right)-\mathbf{U}_{S S}\left(\mathbf{x}-\xi ; \mathbf{e}_{y}, \mathbf{e}_{y}\right)-\mathbf{U}_{S S}\left(\mathbf{x}-\xi ; \mathbf{e}_{z}, \mathbf{e}_{z}\right)\right] d \xi \\
=\alpha_{5} \int_{-c}^{c}\left(c^{2}-\xi^{2}\right) \mathbf{U}_{S S}\left(\mathbf{x}-\xi ; \mathbf{e}_{x}, \mathbf{e}_{x}\right) d \xi, \\
\mathbf{u}_{p g}=\frac{1}{3} \beta_{5} \int_{-c}^{c}\left(c^{2}-\xi^{2}\right)^{2}\left[2 \frac{\partial}{\partial x} \mathbf{U}_{D}\left(\mathbf{x}-\xi ; \mathbf{e}_{x}\right)-\frac{\partial}{\partial y} \mathbf{U}_{D}\left(\mathbf{x}-\xi ; \mathbf{e}_{y}\right)-\frac{\partial}{\partial z} \mathbf{U}_{D}\left(\mathbf{x}-\xi ; \mathbf{e}_{z}\right)\right] d \xi \\
=\beta_{5} \int_{-c}^{c}\left(c^{2}-\xi^{2}\right)^{2} \frac{\partial}{\partial x} \mathbf{U}_{D}\left(\mathbf{x}-\xi ; \mathbf{e}_{x}\right) d \xi, \\
p=\alpha_{5} \int_{-c}^{c}\left(c^{2}-\xi^{2}\right) P_{S S}\left(\mathbf{x}-\xi ; \mathbf{e}_{x}, \mathbf{e}_{x}\right) d \xi
\end{gathered}
$$


In (62a), $\mathrm{U}$ assumes the expression (61), and in $(62 b, c)$, the last expressions are obtained by some rearrangement and using the equation $\nabla^{2}\left(R^{-1}\right)=0$. Carrying out the integrations, we obtain

$$
\begin{array}{r}
\mathbf{u}=2 \mathbf{e}_{x}\left\{\Omega x+2\left(\alpha_{5}+6 \beta_{5}\right) x B_{1,0}-\left(\alpha_{5}+4 \beta_{5}\right)\left(R_{1}-R_{2}\right)\left(3+\frac{c^{2}}{R_{1} R_{2}}\right)+\alpha_{5} c x\left(\frac{1}{R_{1}}+\frac{1}{R_{2}}\right)\right\} \\
-r \mathbf{e}_{r}\left\{\Omega+2\left(\alpha_{5}+6 \beta_{5}\right)\left[B_{1,0}-c\left(\frac{1}{R_{1}}+\frac{1}{R_{2}}\right)\right]\right. \\
\left.-4 \beta_{5}\left[3 x\left(\frac{1}{R_{2}}-\frac{1}{R_{1}}\right)+\left(c^{2}-3 x^{2}\right) B_{3,0}\right]\right\}, \quad(63 a)
\end{array}
$$

in which the notation follows (24). By using (25), we find that $\mathbf{u}$ assumes at the spheroid surface the value

$$
\begin{array}{r}
\mathbf{u}_{0}=2 x \mathbf{e}_{x}\left[\Omega-2 \alpha_{5}\left(3 e-L_{e}\right)-12 \beta_{5}\left(2 e-3 L_{e}\right)\right]-r \mathbf{e}_{r}\left[\Omega+2 \alpha_{5} L_{e}-12 \beta_{5}\left(\frac{2 e}{1-e^{2}}-L_{e}\right)\right] \\
+4 e a^{2}\left(\alpha_{5}-\frac{4 e^{2}}{1-e^{2}} \beta_{5}\right) \frac{\left(1-e^{2}\right) x \mathbf{e}_{x}+r \mathbf{e}_{r}}{a^{2}-e^{2} x^{2}} . \quad(63 b)
\end{array}
$$

From this expression we find that $\mathbf{u}_{0}=0$ if the coefficients of all three terms in $(63 b)$ vanish simultaneously, this being the case if

$$
\alpha_{5}=\frac{4 e^{2}}{1-e^{2}} \beta_{5}=\Omega e^{2}\left[6 e-\left(3-e^{2}\right) \log \frac{1+e}{1-e}\right]^{-1},
$$

which completes our solution. Since the solution involves no Stokeslet and no rotlet, the spheroid experiences in this case neither a net force nor a net moment, as should be expected on the grounds of flow symmetry. This exact solution may be useful in the study of the dynamic stability of a spheroid immersed in an extensional flow. The general case of an extensional flow without axial symmetry can be treated in a similar manner, and incorporation of shear-flow components is possible. Construction of the solution in such a general case is however a little complicated.

In the limit $b \rightarrow a$ (or $e \rightarrow 0$ ), we have

$$
\alpha_{5} \int_{-c}^{c}\left(c^{2}-\xi^{2}\right) d \xi=-\frac{5}{2} \Omega a^{3}, \quad \beta_{5} \int_{-c}^{c}\left(c^{2}-\xi^{2}\right)^{2} d \xi=-\frac{1}{2} \Omega a^{5}
$$

and we deduce that for a sphere of radius $a$ centred in the extensional flow (61)

$$
\mathbf{u} / \Omega=2 x \mathbf{e}_{x}-y \mathbf{e}_{y}-z \mathbf{e}_{z}+\frac{5}{2} a^{3} \frac{\partial}{\partial x}\left(\frac{\mathbf{e}_{x}}{R}+\frac{x \mathbf{x}}{R^{3}}\right)-\frac{1}{2} a^{5} \nabla \frac{\partial^{2}}{\partial x^{2}}\left(\frac{1}{R}\right)
$$

The stream function $\psi(x, r)$, defined by

$$
r u=\partial \psi / \partial r, \quad r u_{r}=-\partial \psi / \partial x \quad\left(u_{r}=\mathbf{u} . \mathbf{e}_{r}\right)
$$

of the extensional flow past a sphere is easily obtained by integration from $(66 a)$ :

$$
\psi=\Omega x\left(y^{2}+z^{2}\right)\left[1-\frac{5}{2}\left(\frac{a}{R}\right)^{3}+\frac{3}{2}\left(\frac{a}{R}\right)^{5}\right]
$$

The streamlines of the flow in the plane $z=0$ are shown in figure 4 for several values of $\psi / \Omega a^{3}$. 


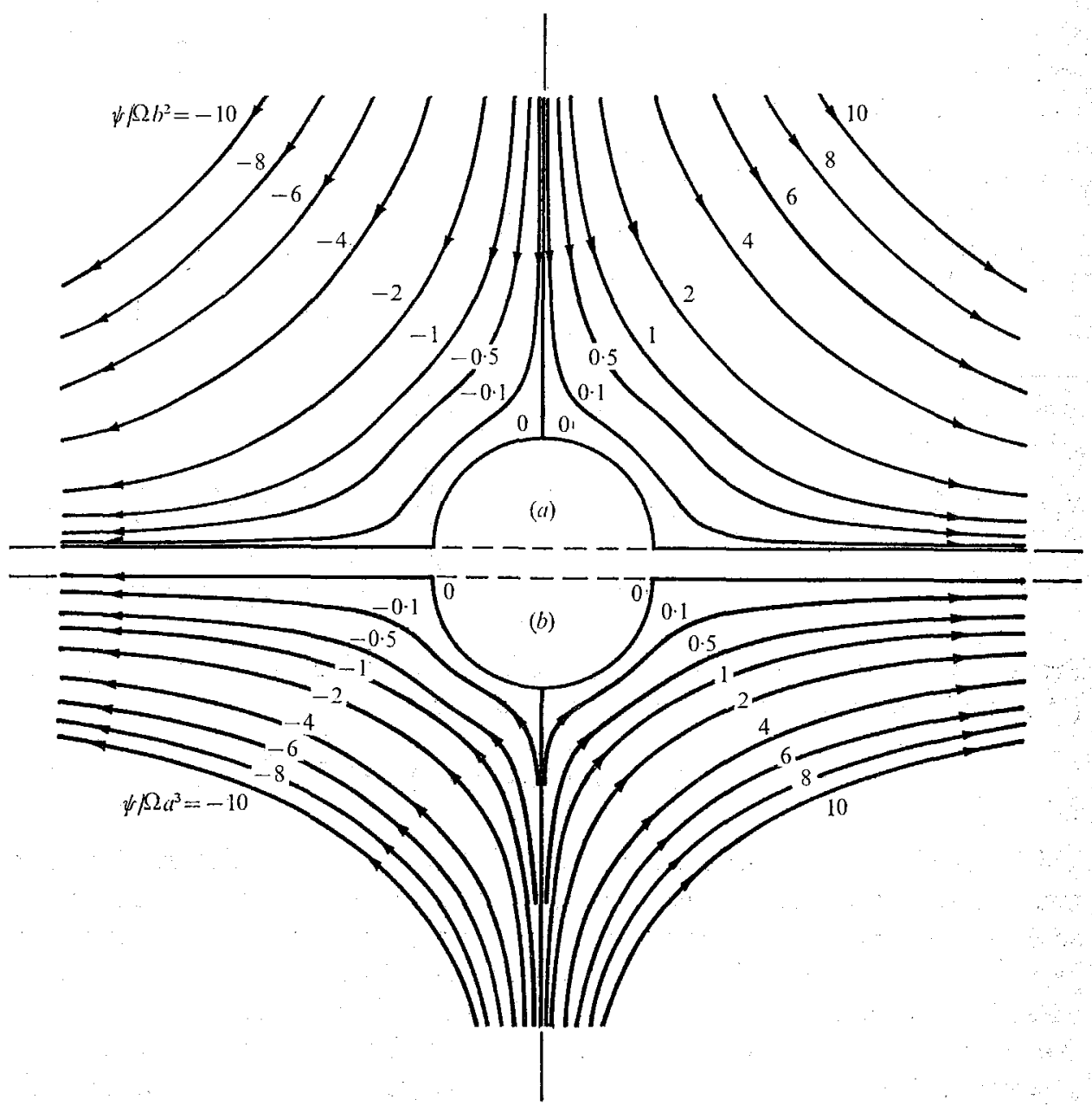

Figure 4. (a) Streamlines of an extensional flow past a circular cylinder of radius $b$. (b) Streamlines in a meridian plane of an axisymmetric extensional flow past a sphere of radius $a$.

\section{Flow with a paraboloidal profile past a sphere}

As a further variation of the free stream, we consider a flow with a paraboloidal velocity profile $\mathrm{U}=K\left(y^{2}+z^{2}\right) \mathbf{e}_{x}$ past a sphere of radius $a$, centred at the origin. (An off-centred paraboloidal profile is equivalent to a centred one superimposed on a uniform flow plus a shear flow.) By the singularity method we find that the solution consists of, apart from the primary flow, a Stokeslet (required to produce a drag), a potential doublet (associated with the Stokeslet to account for the body-thickness effect), an axial Stokes quadrupole (as suggested by the variable velocity gradient) and a potential octupole (associated with the Stokes quadrupole to balance the power-law variations of the solution in $R$ ). Thus we obtain

$$
\mathbf{u}=K r^{2} \mathbf{e}_{x}-\left(C_{1}-C_{2} \frac{\partial^{2}}{\partial x^{2}}\right)\left(\frac{\mathbf{e}_{x}}{R}+\frac{x \mathbf{x}}{R^{3}}\right)+\left(C_{3}-C_{4} \frac{\partial^{2}}{\partial x^{2}}\right) \nabla \frac{\partial}{\partial x}\left(\frac{1}{R}\right),
$$



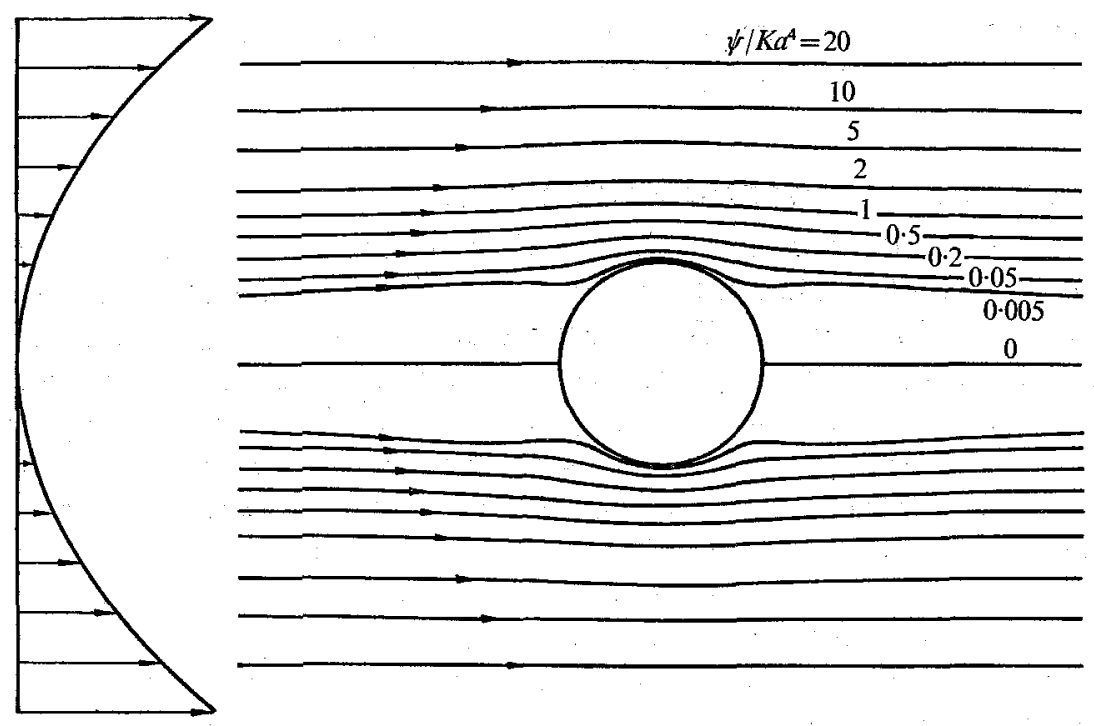

Figure 5. Streamlines in a meridian plane of a paraboloidal flow $U=K\left(y^{2}+z^{2}\right) \mathbf{e}_{x}$ past a sphere of radius $a$.

which is found to vanish at $R=a$ if

$$
C_{1}=\frac{1}{2} K a^{3}, \quad C_{2}=\frac{7}{24} K a^{5}, \quad C_{3}=\frac{5}{12} K a^{5}, \quad C_{4}=\frac{1}{24} K a^{7},
$$

as may be easily verified. The corresponding pressure is given by

$$
p=4 \mu K x+\mu K a^{3}\left(1-\frac{7}{12} a^{2} \frac{\partial^{2}}{\partial x^{2}}\right) \frac{x}{R^{3}}
$$

in which the first term on the right-hand side is the dominant pressure due to the primary flow. The resultant flow has a stream function [defined by (67)] given by

$$
\frac{\psi}{K a^{4}}=\frac{1}{4} r^{4}\left(1-\frac{7}{2} \frac{1}{R^{5}}+\frac{5}{2} \frac{1}{R^{7}}\right)-\frac{1}{2} \frac{r^{2}}{R}\left(1-\frac{1}{R^{2}}\right)^{2},
$$

in which the $R$ and $r$ on the right-hand side have been non-dimensionalized with respect to the sphere radius $a$. Several streamlines in the plane $z=0$ are shown in figure 5. The present solution agrees with the result of Simha (1936).

To compute the sphere drag in this case we first note that although the primary flow has a pressure gradient, hence producing a 'buoyancy' effect (in the negative- $x$ direction) on the sphere, this buoyancy force must be balanced by the net effect of the viscous stress of the primary flow, as must be the case for an arbitrary fluid bulk moving with the primary flow. The drag on the sphere therefore comes solely from the contribution of the Stokeslet:

$$
\mathbf{D}=8 \pi \mu C_{1} \mathbf{e}_{x}=4 \pi \mu K a^{3} \mathbf{e}_{x}
$$

This drag may be regarded as associated with that on the same sphere in a uniform flow of an equivalent velocity

$$
U_{e}=\frac{2}{3} K a^{2} .
$$


It is of interest to note that this $U_{e}$ is precisely the surface average of the primary flow velocity $U=K r^{2}$ over a spherical surface $R=a$. This rule may perhaps have a general validity for arbitrary non-uniform flows.

\section{Centred shear flow past a circular cylinder}

We proceed to discuss some solutions that can exist in the case of unbounded two-dimensional Stokes flows. In contrast with the well-known 'Stokes paradox' for a cylinder placed in a uniform flow, we observe here that, if an unbounded primary flow, which is necessarily non-uniform, and the body geometry are fitted together in such a way as to produce no net force on the body, then a solution, in the strict sense of the Stokes approximation, may be possible.

A simple example of such a situation is the shear flow $U=\Omega z \mathbf{e}_{y}$ past a circular cylinder (of radius $b$ say) fixed at the origin with its axis perpendicular to the stream. In virtue of the flow antisymmetry, we realize that the solution can admit no Stokeslet since there cannot be a net force on the cylinder, but may require a rotlet (in response to the primary vorticity), a stresslet (for counterbalancing the transverse gradient of the shear flow velocity) and a potential quadrupole (which is generally associated with a stresslet). Thus, as a trial solution, we write

$$
\mathbf{u}=\Omega z \mathbf{e}_{y}+\alpha \frac{\mathbf{e}_{x} \times \mathbf{x}}{r^{2}}-\beta \frac{2 y z \mathbf{x}}{r^{4}}-\gamma \nabla \frac{\partial^{2}}{\partial y \partial z}\left(\log \frac{1}{r}\right),
$$

where $\mathbf{x}=y \mathbf{e}_{y}+z \mathbf{e}_{z}, r=|\mathbf{x}|=\left(y^{2}+z^{2}\right)^{\frac{1}{2}}$, and $\alpha, \beta$ and $\gamma$ are the strengths of the rotlet, stresslet and potential quadrupole, in that order. In fact, this expression for $\mathfrak{u}$ is found to satisfy the no-slip condition at $r=b$ if

$$
\alpha=\frac{1}{2} \Omega b^{2}, \quad \beta=\Omega b^{2}, \quad \gamma=\frac{1}{4} \Omega b^{4} .
$$

The corresponding stream function $\psi(y, z)$, defined by $v=\partial \psi / \partial z$ and $w=-\partial \psi / \partial y$, is given by

$$
\frac{\psi}{\Omega b^{2}}=\frac{1}{2}\left(\frac{z}{b}\right)^{2}\left(1-\frac{b^{2}}{r^{2}}\right)^{2}+\frac{1}{4}\left(1-\frac{b^{2}}{r^{2}}\right)-\frac{1}{2} \log \frac{r}{b} .
$$

As shown in figure 6, the flow has two 'backflow' regions, which are symmetric about the $z$ axis and bounded by streamlines that separate from the circular cylinder at

$$
r=b \text { and } \tan ^{-1}(z / y)= \pm 30^{\circ}
$$

(at which four points $\partial^{2} \psi / \partial y^{2}=\partial^{2} \psi / \partial z^{2}=0$ ). This strong upstream and downstream 'blocking effect' is characteristic of the two-dimensional flow; such a backflow feature is absent, for instance, in the shear flow past a sphere given by (49).

The moment on the cylinder (per unit breadth in the $x$ direction), by (9) and (71), is

$$
\mathbf{M}=-4 \pi \mu \alpha \mathbf{e}_{x}=-2 \pi \mu \Omega b^{2} \mathbf{e}_{x} .
$$

If the circular cylinder so located in the shear flow is allowed to rotate freely, its steady angular velocity will be $-\Omega_{0} \mathbf{e}_{x}$, where

$$
\Omega_{0}=\frac{1}{2} \Omega \text {, }
$$



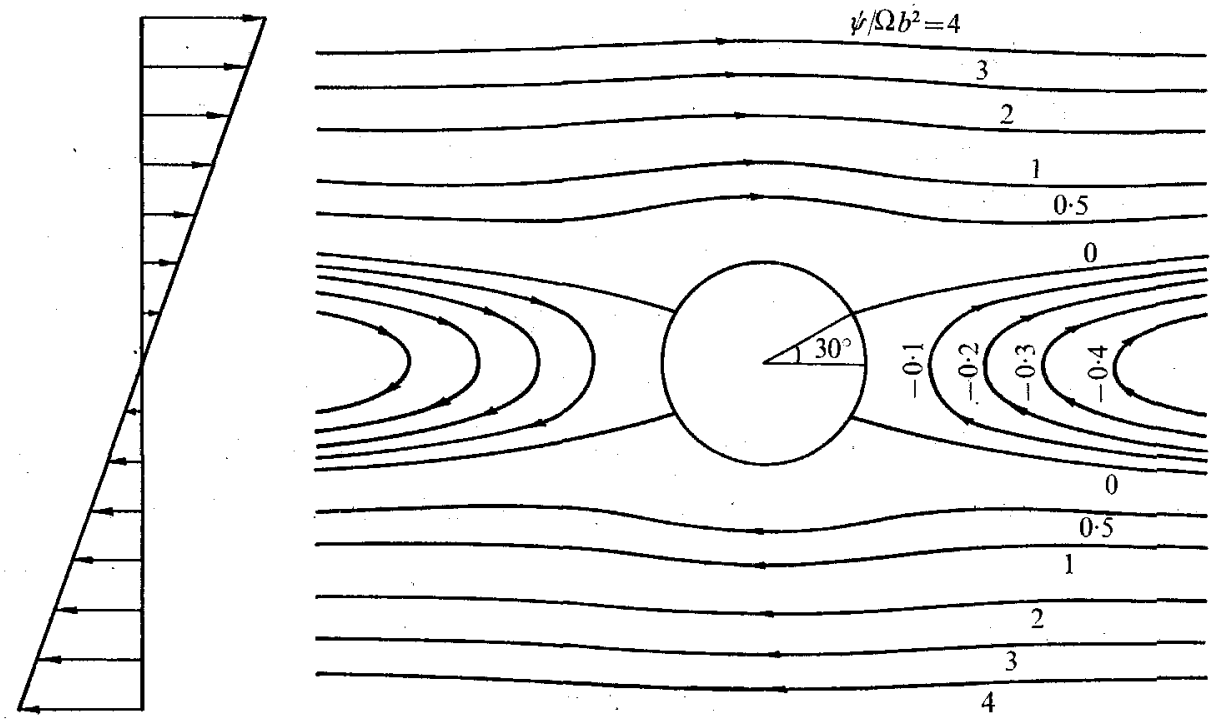

Figure 6. Streamlines of a centred shear flow $\left(\mathbf{U}=\Omega y \mathbf{e}_{x}\right)$ past a circular cylinder of radius $b$. There exist two 'backflow' regions, which are bounded by separation streamlines intersecting the cylinder at angular positions of $\pm 30^{\circ}$ as shown. These separation positions are independent of the shear rate of the primary flow.

since the moment on the cylinder when rotating at angular velocity $\Omega_{0}$ in a viscous fluid otherwise at rest is $\mathbf{M}_{0}=4 \pi \mu \Omega_{0} b^{2} \mathbf{e}_{x}$ and we must have $\mathbf{M}+\mathbf{M}_{0}=0$ for a freely rotating cylinder.

It is essential to note that the present solution exists primarily owing to the particular feature that no Stokeslet is involved in the solution. This feature is violated, for instance, when $U(z)$ is off-centre, so that $\mathbf{U} \neq 0$ along the streamline passing through the centre of the cylinder.

\section{Two-dimensional extensional flow past a circular cylinder}

As another example of possible two-dimensional Stokes flows we consider an extensional flow $\mathbf{U}=\Omega\left(y \mathbf{e}_{y}-z \mathbf{e}_{z}\right)$ past a circular cylinder of radius $b$ centred at the origin. Construction of the solution is analogous to the three-dimensional case already discussed in $\S 8$. It is easily verified that the required solution is

$$
\mathbf{u}=\Omega\left(y \mathbf{e}_{y}-z \mathbf{e}_{z}\right)+A \frac{\partial}{\partial y}\left(\mathbf{e}_{y} \log \frac{1}{r}+\frac{y \mathbf{x}}{r^{2}}\right)-B \nabla\left[\frac{\partial^{2}}{\partial y^{2}}\left(\log \frac{1}{r}\right)\right]
$$

with

$$
A=2 \Omega b^{2}, \quad B=\frac{1}{2} \Omega b^{4} .
$$

Here $\frac{1}{2} A$ is the strength of the two original stresslets and $\frac{1}{2} B$ that of the two original potential quadrupoles, before they are combined to yield the above simplified expression. The two-dimensional stream function $\psi(y, z)$ of the flow is given by

$$
\frac{\psi}{\Omega b^{2}}=y z\left(1-\frac{b^{2}}{r^{2}}\right)^{2},
$$

the corresponding streamlines being shown in figure 4 together with the sphere case for comparison. 


\section{Flow between two confocal prolate spheroids in axial rotation}

The singularity method can be further extended to consider interior flow problems by admitting also the fundamental solutions for interior flows, such as rotons, Stokesons, and so forth.

As the first example of this category we consider the flow between two confocal prolate spheroids

$$
x^{2} / a_{i}^{2}+r^{2} / b_{i}^{2}=1 \quad\left(i=1,2, a_{i} \geqslant b_{i}, a_{2}>a_{1}\right)
$$

with a common focal length $2 c$ and eccentricities $e_{1}$ and $e_{2}$,

$$
c^{2}=a_{1}^{2}-b_{1}^{2}=a_{2}^{2}-b_{2}^{2}, \quad c=e_{1} a_{1}=e_{2} a_{2} \quad\left(e_{1}>e_{2}\right),
$$

which are kept rotating about their major axes with angular velocities

$$
\boldsymbol{\Omega}_{1}=\Omega_{\mathbf{1}} \mathbf{e}_{x}, \quad \mathbf{\Omega}_{\mathbf{2}}=\Omega_{\mathbf{2}} \mathbf{e}_{x},
$$

respectively. The no-slip condition now requires that

$$
\mathbf{u}=\boldsymbol{\Omega}_{i} \times \mathbf{x} \quad\left(\mathbf{x} \text { on } S_{i}, i=1,2\right),
$$

where $S_{1}$ and $S_{2}$ designate the inner and outer body surfaces.

We find that the velocity has the following representation:

$$
\mathbf{u}=\alpha_{0} \mathbf{e}_{x} \times \mathbf{x}+\beta_{0} \int_{-c}^{c}\left(c^{2}-\xi^{2}\right) \mathbf{U}_{R}\left(\mathbf{x}-\xi ; \mathbf{e}_{x}\right) d \xi .
$$

The roton, of undetermined strength $\alpha_{0}$, is required since the interior flow must reduce to a solid-body rotation when the inner spheroid vanishes. The line distribution of rotlets, with a parabolic density between the two foci, is retained from the single-spheroid case (see part 1), to which the present solution must reduce as the outer spheroid recedes to infinity. Now, on $S_{1}$ and $S_{2},(77)$ becomes

$$
\mathbf{u}_{i}=\left[\alpha_{0}+\beta_{0} f\left(e_{i}\right)\right]\left(\mathbf{e}_{x} \times \mathbf{x}\right) \quad\left(\mathbf{x} \text { on } S_{i}, i=1,2\right),
$$

where

$$
f(e)=\frac{2 e}{1-e^{2}}-\log \frac{1+e}{1-e} \text {. }
$$

Hence the no-slip conditions (76) are satisfied if

$$
\alpha_{0}=\frac{\Omega_{2} f_{1}-\Omega_{1} f_{2}}{f_{1}-f_{2}}, \quad \beta_{0}=\frac{\Omega_{1}-\Omega_{2}}{f_{1}-f_{2}} \quad\left(f_{i} \equiv f\left(e_{i}\right)\right) .
$$

The moment acting on the inner spheroid, according to (9), is

$$
\mathbf{M}=-8 \pi \mu \beta_{0} \mathbf{e}_{x} \int_{-c}^{c}\left(c^{2}-\xi^{2}\right) d \xi=-\frac{32}{3} \pi \mu c^{3} \frac{\Omega_{1}-\Omega_{2}}{f_{1}-f_{2}} \mathbf{e}_{x}
$$

which is equal and opposite to the moment on the outer spheroid since there exists no other extraneous moment.

We note here that the rotlet strength, and hence also the moment $M$, will vanish either when $\Omega_{1}=\Omega_{2}$ or when the inner spheroid shrinks to a line $\left(b_{1} \rightarrow 0, e_{1} \rightarrow 1\right)$, for in either case the flow is reduced to a solid-body rotation. When the outer spheroid recedes to infinity $\left(a_{2} \rightarrow \infty, e_{2} \rightarrow 0\right)$, the present solution reduces to the single-spheroid case already treated in part 1 . 
In the limiting case of two concentric spheres $\left(e_{1} \rightarrow 0\right.$ and $e_{2} \rightarrow 0$ for fixed $a_{1}$ and $\left.a_{2}\right),(78)$ reduces to the known solution (e.g. Landau \& Lifshitz 1959, p. 69), while $(80 a)$ becomes

$$
\mathbf{M}=-8 \pi \mu\left(\Omega_{1}-\Omega_{2}\right)\left(a_{1}^{-3}-a_{2}^{-3}\right)^{-1} \mathbf{e}_{x}
$$

\section{Flow between two concentric spheres in relative translation}

As another example of interior Stokes flow we consider the relative translation of two spheres at the instant when the two centres coincide. Under the Stokes approximation, we may choose, with no loss of generality, the inner sphere to be fixed while the outer moves with velocity $\mathbf{U}=U \mathbf{e}_{x}$ (which may be regarded as the relative velocity when both spheres are in translation). The no-slip condition then requires that

$$
\mathbf{u}=0 \quad\left(R=a_{1}\right), \quad \mathbf{u}=U \mathbf{e}_{x} \quad\left(R=a_{2}\right),
$$

$a_{1}$ and $a_{2}$ being the radii of the two spheres $\left(a_{1}<a_{2}\right)$.

The velocity is found to consist of a uniform flow, a Stokeslet, a potential doublet and a Stokeson and is of the form

$$
\mathbf{u}=U_{1} \mathbf{e}_{x}-B_{1}\left(\frac{\mathbf{e}_{x}}{R}+\frac{x \mathbf{x}}{R^{3}}\right)+B_{2} \nabla \frac{\partial}{\partial x}\left(\frac{1}{R}\right)-B_{3}\left(2 R^{2} \mathbf{e}_{x}-x \mathbf{x}\right),
$$

the corresponding pressure being

$$
p=-2 \mu B_{1} x / R^{3}-10 \mu B_{3} x,
$$

in which the last term represents the prevalent Stokeson pressure. In fact, the above expression for $\mathbf{u}$ satisfies the no-slip conditions (81), and hence is the solution sought, if, as can be readily verified,

$$
\begin{gathered}
U_{1}=U \sigma\left(1+\frac{5}{4} \lambda^{3}-\frac{9}{4} \lambda^{5}\right), \quad B_{1}=\frac{3}{4} U a_{1} \sigma\left(1-\lambda^{5}\right), \\
B_{2}=\frac{1}{4} U a_{1}^{3} \sigma\left(1-\lambda^{3}\right), \quad B_{3}=\frac{3}{4} U a_{1}^{-2} \sigma \lambda^{3}\left(1-\lambda^{2}\right) \\
\lambda=a_{1} / a_{2} \quad(<1), \quad \sigma^{-1}=(1-\lambda)^{4}\left(1+\frac{7}{4} \lambda+\lambda^{2}\right) .
\end{gathered}
$$

where

This solution has been obtained previously (for the references see Happel \& Brenner 1965, p. 130) by the stream-function method. The drag on the inner sphere is simply

$$
\mathbf{F}=8 \pi \mu B_{1} \mathbf{e}_{x}=6 \pi \mu U a_{1} \sigma\left(1-\lambda^{5}\right) \mathbf{e}_{x}
$$

which is of course equal and opposite to the force on the outer sphere according to the principle of action and reaction. As shown in figure 7, the drag recovers the single-sphere value as $\lambda \rightarrow 0$, and increases rapidly as $\lambda=a_{1} / a_{2} \rightarrow 1$ like

$$
F^{\prime} / 6 \pi \mu U a_{1}=\frac{4}{3}(1-\lambda)^{-3}\left[1-(1-\lambda)+O(1-\lambda)^{2}\right] .
$$

Although the present solution applies only to the instant when the two spheres become concentric, it nevertheless provides a valuable guideline as to the importance of wall effects and provides an estimate of results for particle interactions in rheology. It can further be applied to the problem of small oscillations of two concentric spheres. 


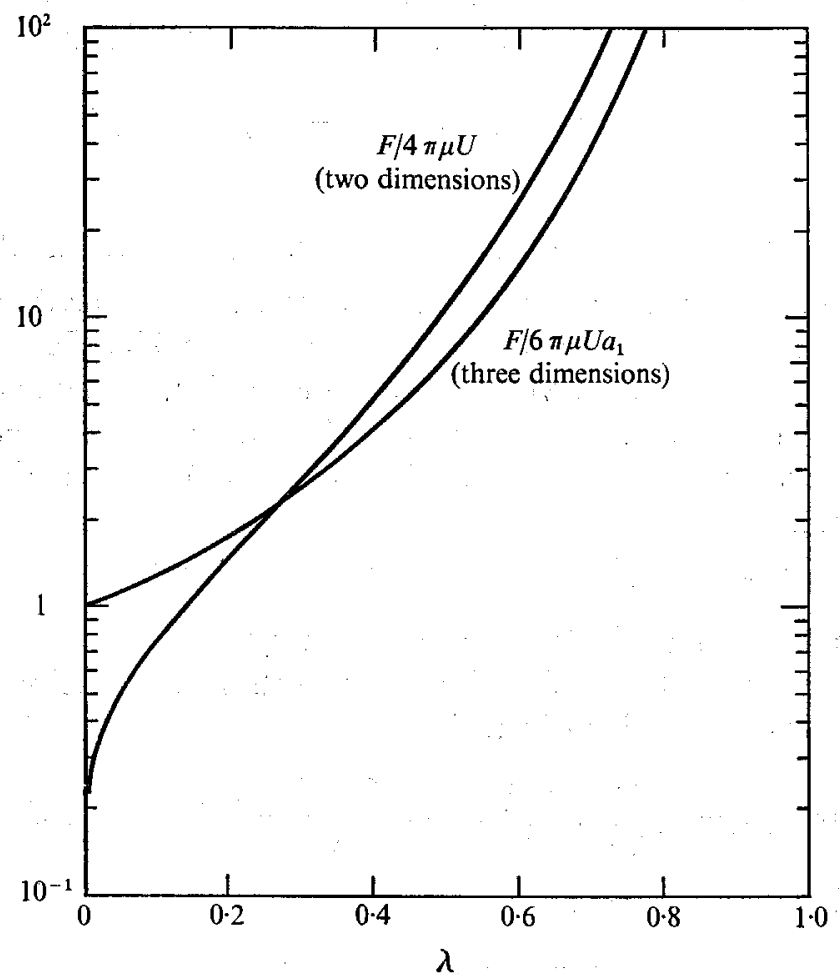

Figure 7. Variations of the forces on two concentric cylinders (of radii $b_{1}$ and $b_{2}$ ) and two concentric spheres (of radii $a_{1}$ and $\left.a_{2}\right) v s$. their radius ratio $\lambda\left(\lambda=b_{1} / b_{2}=a_{1} / a_{2}\right)$.

\section{Flow between two concentric cylinders in relative translation}

Regarding still another aspect of the Stokes paradox, we observe that the solution for two-dimensional Stokes flow involving a translating cylinder may exist if the flow is bounded in all transverse directions. The simplest problem in this category is the relative translation of two concentric cylinders. In complete analogy with the concentric-spheres problem, we let the inner cylinder $\left(r=b_{1}\right.$, $\left.r^{2}=y^{2}+z^{2}\right)$ be fixed while the outer cylinder $\left(r=b_{2}\right)$ moves with velocity $\mathrm{U}=U \mathbf{e}_{y}$. The velocity and pressure can be written as

$$
\begin{aligned}
\mathbf{u} & =U_{2} \mathbf{e}_{y}-C_{1}\left(\mathbf{e}_{y} \log \frac{b_{1}}{r}+\frac{y \mathbf{x}}{r^{2}}\right)+C_{2}\left(-\frac{\mathbf{e}_{y}}{r^{2}}+\frac{2 y \mathbf{x}}{r^{4}}\right)-C_{3}\left(3 r^{2} \mathbf{e}_{y}-2 y \mathbf{x}\right), \\
p & =-2 \mu C_{1} y / r^{2}-8 \mu C_{3} y,
\end{aligned}
$$

in which the fundamental singularities, as well as $\mathbf{x}$, all assume their twodimensional forms in the $y, z$ plane. The no-slip boundary conditions $(\mathbf{u}=0$ at $r=b_{1}, \mathbf{u}=U \mathbf{e}_{y}$ at $r=b_{2}$ ) are satisfied if

$$
\begin{array}{ll}
U_{2}=\frac{1}{2} U \sigma\left(1+3 \lambda^{2}\right), & C_{1}=U \sigma\left(1+\lambda^{2}\right), \\
C_{2}=\frac{1}{2} U \sigma b_{1}^{2}, & C_{3}=\frac{1}{2} U \sigma b_{1}^{-2} \lambda^{2},
\end{array}
$$


where

$$
\lambda=b_{1} / b_{2}, \quad \sigma^{-1}=\left(1+\lambda^{2}\right) \log (1 / \lambda)-\left(1-\lambda^{2}\right) .
$$

The stream function of the flow can be deduced from (85a) as

$$
\frac{\psi}{U b_{1}}=\frac{1}{2} \frac{z}{b_{1}} \sigma\left[2\left(1+\lambda^{2}\right)\left(\log \frac{r}{b_{1}}-1\right)+1+3 \lambda^{2}+\left(\frac{b_{1}}{r}\right)^{2}-\left(\frac{r}{b_{1}}\right)^{2} \lambda^{2}\right] .
$$

The force on the inner cylinder, by (20), is

$$
\mathbf{F}=4 \pi \mu C_{1} \mathbf{e}_{y}=4 \pi \mu U \sigma\left(1+\lambda^{2}\right) \mathbf{e}_{y} .
$$

As shown in figure 7 , the drag increases drastically as $\lambda=b_{1} / b_{2}$ approaches unity, like

$$
F / 4 \pi \mu U=3(1-\lambda)^{-3}\left[1-\frac{3}{2}(1-\lambda)+O(1-\lambda)^{2}\right] .
$$

Of particular interest is the limiting case when the outer cylinder is very large compared with the inner one $\left(\lambda=b_{1} / b_{2} \ll 1\right)$, in which case the drag becomes

$$
F=\frac{4 \pi \mu U}{\log (1 / \lambda)-1}\left[1-\frac{2 \lambda^{2}}{\log (1 / \lambda)-1}+O\left(\frac{\lambda^{4}}{\log \lambda}\right)\right] .
$$

This result seems to suggest that $F$ would diminish logarithmically as $b_{2} / b_{1} \rightarrow \infty$. It should be emphasized, however, that the present solution based on the Stokesflow approximation can be valid only when $U b_{1} / \nu \ll 1$ as well as $U b_{2} / \nu \ll 1$.

\section{Conclusions}

We have presented here the exact solutions to a number of exterior and interior Stokes-flow problems involving prolate spheroids, spheres and circular cylinders and a variety of primary flows. Although some of these solutions were known, as cited in the text in specific cases, their representations in terms of distributed singularities are believed to be new. They further demonstrate the effectiveness of the method and begin to provide a physical picture of various singularities pertaining to body geometries and flows. This physical feeling has led us to construct simply new solutions to a few more challenging problems here.

A primary objective of the present study is to throw light on further development of the singularity method for bodies of arbitrary shape, which may depend on the time as in micro-organism locomotion, and for arbitrary primary flows, which may appear under various circumstances. It is in this respect useful to expound upon the mathematical technique for ascertaining 'suitable singularities' as well as their distributions. For this purpose we summarize in table 1 the salient features of the singularities and the physical roles they play in the exact solutions for a prolate spheroid immersed in different primary flows. (In table $1, \mathbf{U}$ denotes the primary-flow velocity, $\zeta_{0}$ its vorticity, $\mathbf{e}_{i}(i=1,2,3)$ the base vectors along the spheroid's principal axes, $\mathbf{e}_{1}$ coinciding with the longitudinal axis of revolution, $\epsilon_{i j k}$ is the alternating tensor and $\alpha_{i}, \beta_{i}$ and $\gamma_{i}$ are constants.)

In general, these exact solutions seem to suggest the following rules for other axisymmetric bodies in arbitrary flows. 
Primary flow

$$
\begin{gathered}
\text { Uniform } \\
\mathbf{U}=\mathbf{e}_{\boldsymbol{i}}
\end{gathered}
$$

Centred shear

$$
\begin{aligned}
& \mathbf{U}=x_{j} \mathbf{e}_{i} \quad(i \neq j) \\
& \zeta_{0}=-\mathbf{e}_{k} \epsilon_{i j k}
\end{aligned}
$$

Extensional

$$
\begin{aligned}
& \mathbf{U}=2 x_{1} \mathbf{e}_{1}-x_{2} \mathbf{e}_{2}-x_{3} \mathbf{e}_{3}\left\{\begin{array}{c}
\text { stresslet } \\
+ \text { quadrupole }
\end{array}\right. \\
& \zeta_{0}=0
\end{aligned}
$$

Paraboloidal flow (past a sphere)

$\mathbf{U}=\left(x_{2}^{2}+x_{3}^{2}\right) \mathbf{e}_{1}$

$\zeta_{0}=2\left(x_{3} \mathrm{e}_{2}-x_{2} \mathrm{e}_{3}\right)$

$$
\left\{\begin{array}{r}
\text { Stokeslet } \\
+ \text { doublet }
\end{array}\right.
$$

$\left\{\begin{array}{c}\text { rotlet } \\ + \text { stresslet } \\ \text { + quadrupole }\end{array}\right.$

\section{Stokeslet}

+ doublet

\section{Singularities}

$\{+$ Stokes quadrupole

$$
\begin{array}{r}
-\mathbf{U}_{S}\left(\mathbf{x}-\xi ; \mathbf{e}_{i}\right) \\
\mathbf{U}_{D}\left(\mathbf{x}-\xi ; \mathbf{e}_{i}\right)
\end{array}
$$$$
\mathbf{U}_{R}\left(\mathbf{x}-\xi ; \mathbf{e}_{k}\right)
$$$$
\mathbf{U}_{S S}\left(\mathbf{x}-\xi ; \mathbf{e}_{i}, \mathbf{e}_{j}\right)
$$$$
\partial \mathbf{U}_{D}\left(\mathbf{x}-\xi ; \mathbf{e}_{i}\right) / \partial x_{j}
$$

$$
\mathbf{U}_{S S}\left(\mathbf{x}-\xi ; \mathbf{e}_{1}, \mathbf{e}_{1}\right)
$$$$
\partial \mathbf{U}_{D}\left(\mathbf{x}-\xi ; e_{1}\right) / \partial x_{1}
$$$$
-\mathbf{U}_{S}\left(\mathbf{x}, \mathbf{e}_{\mathbf{1}}\right)
$$$$
\mathbf{U}_{D}\left(\mathbf{x}, \mathbf{e}_{\mathbf{1}}\right)
$$$$
\partial^{2} \mathbf{U}_{S}\left(\mathbf{x}, \mathbf{e}_{1}\right) / \partial x_{1}^{2}
$$$$
-\partial^{2} U_{D}\left(\mathbf{x}, \mathbf{e}_{1}\right) / \partial x_{1}^{2}
$$

Distribution

$(|\xi| \leqslant c)$

$\alpha_{i}$

$\beta_{i}\left(c^{2}-\xi^{2}\right)$

$\gamma_{k}\left(c^{2}-\xi^{2}\right)$

$\alpha_{k}\left(c^{2}-\xi^{2}\right)$

$\beta_{k}\left(c^{2}-\xi^{2}\right)^{2}$

$\alpha\left(c^{2}-\xi^{2}\right)$

$\beta\left(c^{2}-\xi^{2}\right)^{2}$

All at centre of sphere

Table 1

(i) A Stokeslet is required when the primary-flow velocity has a non-zero average over the body surface.

(ii) A rotlet is required when the primary-flow vorticity has a non-zero average over the body surface.

(iii) A stresslet may be required when the primary flow has a velocity gradient with a non-zero average over the body surface. (A stresslet $U_{S S}$ associated with $\mathbf{e}_{i}$ and $\mathbf{e}_{j}$ is suggested for each non-zero surface average of $\partial U_{i} / \partial x_{j}$.)

(iv) A doublet is associated, as a rule, with a Stokeslet, a potential quadrupole with a stresslet, and likewise for higher-order poles, such that in each case the congruent pair have the same dependence on the base vectors $\mathbf{e}_{i}, \mathbf{e}_{j}$ and $\mathbf{e}_{k}$.

(v) For interior Stokes flows, a Stokeson is generally associated with a Stokeslet, a roton with a rotlet, and a stresson with a stresslet.

These rules should be regarded, at this stage, merely as a useful guideline. A precise statement of the necessary and sufficient conditions for their validity, and hence the converse to these rules, will require a mathematical proof, which is under investigation.

With suitable singularities ascertained for an arbitrary (axisymmetric) body in a certain designated flow, the problem becomes one of determining the singularity distribution, both in magnitude and spatial range, from a set of three coupled functional equations of the first kind as a result of the no-slip boundary condition on the velocity components at the body surface. Since the distribution range is unknown a priori, these integral equations are nonlinear equations, about which little theoretical information is available (except for the special case of slender bodies). This general problem will be discussed in a future study.

This work was partially sponsored by the National Science Foundation, under Grant GK 31161X, and by the Office of Naval Research, under Contract N00014-67-A-0094-0012. 


\section{REFERENCES}

BAtchelor, G. K. $1970 a$ The stress system in a suspension of force-free particles. J. Fluid Mech. 41, 545-570.

BATCHELOR, G. K. $1970 b$ Slender-body theory for particles of arbitrary cross-section in Stokes flow. J. Fluid Mech. 44, 419-440.

Blake, J. R. \& Chwang, A. T. 1974 Fundamental singularities of viscous flow. Part I. The image system in the vicinity of a stationary no-slip boundary. J. Engng Math. 8, 23-29.

Broersma, S. 1960 Viscous force constant for a closed cylinder. J. Chem. Phys. 32, $1632-1635$.

BuRGERs, J. M. 1938 On the motion of small particles of elongated form suspended in a viscous liquid. Chap. III of Second Report on Viscosity and Plasticity. Kon. Ned. Akad. Wet., Verhand. 16, 113-184.

Chwang, A. T. \& WU, T. Y. $1974 a$ Hydromechanics of low-Reynolds-number flow. Part 1. Rotation of axisymmetric prolate bodies. $J$. Fluid Mech. 63, 607-622.

Chwang, A. T. \& WU, T. Y. $1974 b$ A note of potential flow involving prolate spheroids. Schiffstech. 21, 19-31.

Cox, R. G. 1970 The motion of long slender bodies in a viscous fluid. Part 1. General theory. $J$. Fluid Mech. 44, 791-810.

Cox, R. G. 1971 The motion of long slender bodies in a viscous fluid. Part 2. Shear flow. $J$. Fluid Mech. 45, 625-657.

EDWARDES, D. 1892 Steady motion of a viscous liquid in which an ellipsoid is constrained to rotate about a principal axis. Quart. J. Math. 26, 70-78.

GaNs, R. 1928 Zur Theorie der Brownschen Molekularbewegung. Ann. Phys. 86, 628-656.

GraY, J. \& HaNCoCK, G. J. 1955 The propulsion of sea-urchin spermatozoa. J. Exp. Biol. $32,802-814$.

HANCOCK, G. J. 1953 The self-propulsion of microscopic organisms through liquids. Proc. Roy. Soc. A 217, 96-121.

Happel, J. \& Brenner, H. 1965 Low Reynolds Number Hydrodynamics. Prentice-Hall.

JEFFERY, G. B. 1922 The motion of ellipsoidal particles immersed in a viscous fluid. Proc. Roy. Soc. A 102, 161-179.

LAMB, H. 1932 Hydrcdynamics. Cambridge University Press.

Landau, L. D. \& Lifshitz, E. M. 1959 Fluid Mechanics. Pergamon.

LANDWEBER, L. 1951 The axially symmetric potential flow about elongated bodies of revolution. Rep. Taylor Model Basin, no. 761.

LoRENTz, H. A. 1897 A general theorem concerning the motion of a viscous fluid and a few consequences derived from it. Versl. Kon. Akad. Wet. Amst. 5, 168-175.

OrERBECK, A. 1876 Ueber stationäre Flüssigkeitsbewegungen mit Berücksichtigung der inneren Reibung. $J$. reine angew. Math. 81, 62-80.

OseEN, C. W. 1927 Hydrodynamik. Leipzig: Akad. Verlagsgesellschaft.

Sтмна, R. 1936 Untersuchungen über die Viskosität von Suspensionen und Lösungen. Kolloid Z. 76, 16-19.

TAYLOR, G. I. 1969 Motion of axisymmetric bodies in viscous fluids. In Problems of Hydrodynamics and Continuum Mechanics, pp. 718-724. S.I.A.M. Publ.

TrureTt, J.P. K. 1970 Axial and transverse Stokes flow past slender axisymmetric bodies. J. Fluid Mech. 44, 401-417.

Tuck, E. O. 1964 Some methods for flows past blunt slender bodies. J. Fluid Mech. 18, 619-635.

Tuck, E. O. 1970 Toward the calculation and minimization of Stokes drag on bodies of arbitrary shape. Proc. 3rd Austr. Conf. on Hydraulics \& F'luid Mech. pp. 29-32. 